\title{
Shock Boundary Layer Interactions in a Low Aspect Ratio Duct
}

\author{
Laura M. Campo*, John K. Eaton \\ Department of Mechanical Engineering, Stanford University, Stanford CA, 94305, USA
}

\begin{abstract}
Experimental data acquired using high resolution two-component particle image velocimetry (PIV) are presented for shock boundary layer interactions (SBLIs) generated by a compression-expansion ramp geometry. The incident oblique shock wave is generated by a sub-boundary layer height ramp inclined $20^{\circ}$ to the $M_{\infty}=2.05$ inflow. Results are presented for two different ramp sizes $\left(h_{\text {ramp }} / \delta_{0}=0.56\right.$ and $\left.h_{\text {ramp }} / \delta_{0}=0.93\right)$, and compared to a previously documented $h_{\text {ramp }} / \delta_{0}=0.20$ case. For each case, mean velocitiy and turbulent statistics for both the SBLI at the foot of the compression ramp and the incident/reflected SBLI on the opposite wall are analyzed. Data are acquired in several streamwise-vertical planes across the span of the low aspect ratio duct in order to document spanwise non-uniformities and confinement effects, with the specific goal of producing a high quality experimental database for CFD validation. The angles of the incident and reflected shock waves become steeper as the side wall is approached, due to the lower velocities within the side wall boundary layer. Mean flow reversal is observed near the spanwise centerline of the duct, but only instantaneous flow reversal is seen closer to the side walls. These spanwise non-uniformities are more prominent for the stronger interactions caused by the larger ramp geometry. For this case there is no nominally two-dimensional region of the flow, and a Mach stem occurs in the core of the flow, causing a significant subsonic wake. The shock excursion length scale relative to the incoming boundary layer thickness, $L_{e x} / \delta_{0}$, is measured for all of the shock features and found to be significantly lower than values reported in the literature for similar flows. Furthermore, $L_{e x} / \delta_{0}$ for the reflected shock does not depend on the strength of the incident shock or the size of the separated zone.
\end{abstract}

Keywords: shock waves, separated flow, confinement effects, Mach stem, experimental database, validation

\section{Introduction}

Shock wave boundary layer interactions (SBLIs) are important physical phenomena in a variety of applications in supersonic and hypersonic transport. They are associated with shock wave unsteadiness, increased drag and heating, and shock induced boundary layer separation, all of which can have drastic adverse consequences for aerodynamic surfaces and propulsion systems. Many experimental and numerical studies of shock boundary layer interactions in various configurations have been carried out over the past several decades (see e.g. Dolling (2001) or Clemens and Narayanaswamy (2014) for reviews).

A few simple canonical geometries have been used widely in both laboratory experiments and simulations to study various aspects of shock boundary layer interactions. Two of the most common configurations are the compression ramp and the incident/reflected shock geometries (see e.g. Settles and Dodson (1994)). In the compression ramp setup, a supersonic flow encounters an angled wedge which generates an oblique shock wave that deflects the flow around the obstacle. Traditionally the compression ramp is large relative to the incoming boundary layer thickness, meaning that the inviscid shock angle and deflection angle of the freestream are set by the inviscid oblique shock relations. In the incident/reflected shock

*corresponding author, e-mail: lcampo@stanford.edu interaction, an externally generated shock interacts with a wall, disturbing the boundary layer and reflecting from a point above the surface. The incident shock is generally imposed by a sharpedged wedge or angled plate suspended in the freestream flow, again setting up a flow deflection that is known a priori from theory.

The majority of experimental SBLI studies have either focused on a nominally two-dimensional region in the center of a duct (Beresh et al. (2002), Hou et al. (2003), Humble et al. (2007), Dupont et al. (2008), Souverein et al. (2010)) or have intentionally avoided corner and sidewall effects by employing aerodynamic fences and/or non-fully-spanning shock generators in experiments (Settles et al. (1979), Dolling and Or (1985), Andreopoulos and Muck (1987), Erengil and Dolling (1991), Hou et al. (2003), Bookey et al. (2005), Ringuette et al. (2009), Lapsa (2009)). These studies have provided a wealth of knowledge about a nominally two-dimensional region of the flow, including proposed mechanisms for the low frequency unsteadiness of the shock wave, separation and interaction length scales, and the effect of increasing ramp angle on shock strength and boundary layer separation. However, questions remain about how the flow outside this region is affected by corner vortices and confinement by side walls. Several researchers have concluded that the separation bubble size is underpredicted in simulations with spanwise periodic boundary conditions when compared to experiments in which confinement effects cannot be 
completely avoided (Adamson and Messiter (1980), Hadjadj et al. (2010), Pirozzoli et al. (2010)). Furthermore, Touber and Sandham (2009) reported that the size of the separation bubble varied with changes in the spanwise extent of the computational domain even when spanwise periodic boundary conditions were employed. Galbraith et al. (2013) performed compressible RANS computations of a Mach 2.75 incident shock boundary layer interaction in geometries of aspect ratio one and two. They found significant differences in the amount of blockage and boundary layer separation between the two cases. Furthermore, they report that the flow field is highly three dimensional even with an extruded 2D geometry and that a centerline view of the results cannot be considered as representative of a 2D solution even for the larger aspect ratio tunnel. Dussauge et al. (2006) suggests that the low frequency motion of the shock wave may be coupled to or perhaps caused by threedimensional effects and specifically proposes the question of "what is the effect of sidewalls?"

Ganapathisubramani et al. (2007) used planar laser scattering and wide field PIV measurements in streamwise-spanwise planes to investigate low frequency unsteadiness in a Mach 2 SBLI generated by a $20^{\circ}$ compression ramp. The measurement domain focused on a central region of the tunnel span far from the side walls, but instantaneous coherent 3D structures were still identified in this region. Elongated instantaneous regions of high and low momentum in the streamwise direction were found across the span, but these features were not stationary and therefore not visible in the mean velocity field. Humble et al. (2009) used tomographic PIV to investigate the three-dimensional instantaneous structure of an incident shock boundary layer interaction. These measurements provided insight into the organization of vortical structures and the relationship between coherent large scale motions in the incoming boundary layer with the shock motion. However, similar to the study by Ganapathisubramani, the measurement domain did not extend into a region affected by the side wall boundary layers and corner/sidewall effects were not specifically addressed.

Recently a handful of studies have shifted attention toward directly investigating three-dimensionality induced by side walls and corner effects in SBLI flows. Gaitonde et al. (2001) used a Reynolds-averaged compressible Navier Stokes solver with a $\mathrm{k}-\epsilon$ turbulence model to investigate the sidewall interaction in a simulated scramjet inlet configuration. With appropriate tuning of a limiting term on turbulence energy production, the simulation results agreed qualitatively with the experimental oil flow visualizations of Zheltovodov et al. (1994); however more rigorous quantitative comparisons were not possible. The study concluded that the SBLI flowfield was highly sensitive to the state of the sidewall boundary layers. Hanada et al. (2005) investigated a three-dimensional normal shock interaction in a duct with inlet Mach number of 1.68. Using laser induced fluorescence measurements in several streamwise-spanwise planes, they found that the Mach number immediately downstream of the initial shock wave was highly non-uniform over the cross section, with a higher Mach number near the corner regions than in the core of the flow. Burton and Babinsky (2012) studied corner separation for a normal shock boundary layer interaction at Mach 1.5 using oil flow visualization, laser Doppler anemometry, pitot probe traverses, and pressure sensitive paint. They were able to alter the corner separations using suction and micro-vortex generators and observed that the degree of separation near the centerline increased with decreasing corner separation and vice versa.

Eagle (2012) added to the understanding of 3D separation in a Mach 2.75 incident oblique SBLI using stereo PIV and oil flow visualization. These measurements indicated significant three-dimensionality throughout the interaction, and that no regions of the flow could be accurately represented as nominally two-dimensional. Helmer (2011) \& Helmer et al. (2012) initiated the study upon which the current work expands, making the first high resolution 2D PIV measurements inside the sidewall boundary layer of an oblique SBLI. Bermejo-Moreno et al. (2014) performed wall-modeled LES of the test geometries presented in this paper, using the present experimental database ( $h_{\text {ramp }} / \delta_{0}=0.20$ and 0.56 cases) for validation. They compared simulations performed with spanwise periodic boundary conditions to simulations including no-slip side walls and noted drastic differences in the SBLI features, including the degree of boundary layer separation and whether or not a Mach stem was present.

Dolling (2001) suggested that future SBLI studies should focus on three-dimensional flows and detailed high quality measurements suitable for CFD validation. Settles and Dodson (1994) recommended focusing experimental studies on a variety of points including turbulence data, non-intrusive flow field data for both mean and fluctuating velocities, and more complex types of building block experiments. The study described in this paper is designed specifically for the purpose of CFD validation with these points in mind. The experiment includes measurements of the mean and turbulent velocity fields in both the core of the SBLI flow as well as within the sidewall boundary layers. Experiments are performed in a low aspect ratio $(A R \approx 1)$ duct with a fully spanning shock-generating ramp mounted to the top wall. These features are specifically selected so that confinement effects and spanwise non-uniformities in flow features due to interaction of the shock wave with the sidewall boundary layers can be documented. In all test cases the shock generating ramp has a height smaller than the incoming boundary layer thickness. This geometry causes a coupling between the viscous effects in the SBLI near the compression ramp foot and the freestream flow deflection; e.g. the shock strength can be increased by increasing the ramp size while holding its angle constant. Several previous studies have altered the strength of the shock wave using shock generators of varying angles (see e.g. Settles et al. (1979), Dupont et al. (2006), Souverein et al. (2010)), but to the authors' knowledge this is the first study that achieves the strengthening or weakening effect by changing ramp height at constant angle. Measurements are made both in the vicinity of the compression ramp where the shock is generated as well as on the opposite wall where the shock wave impinges and reflects, allowing both types of interactions to be investigated in a single test case.

An expansion fan emanates from the end of the compression ramp where the wall turns back to horizontal, adding complex- 
ity to the flowfield. This feature is not typical of many compression ramp or incident shock interaction geometries, and most studies aim to delay the expansion fan far downstream to a point where it has no interaction with the primary SBLI of interest. However, the compression-expansion ramp configuration has practical significance to supersonic inlets and has been studied experimentally and numerically (Zheltovodov (1996), Loginov et al. (2007), Grilli et al. (2013)). Hunter Jr. and Reeves (1971) investigated the effect of compression ramp length on separation in compression-expansion ramp configurations and found that for short enough ramps (or small enough $h_{\text {ramp }} / \delta_{0}$ ), the upstream influence of the expansion fan affects flow properties over the ramp. This effect is important in the current study - as the ramp size increases, the expansion fan occurs further downstream, delaying its effects on both the compression ramp and incident shock interactions.

The combination of these unique features makes this experiment a slightly more complex "building block" case, while still allowing for the inlet and boundary conditions to be accurately controlled and documented as is necessary for any dataset which is intended for CFD validation. Wide field mean and fluctuating measurements of the streamwise and vertical velocity components are provided at several stations across the span of the tunnel, allowing for detailed assessment of a simulation's ability to capture the relevant physics in many different regions of the flow. The simple low aspect ratio test section with side walls can be simulated at a lower computational cost than a fully three-dimensional simulation of a higher aspect ratio geometry or one with more complex features like aerodynamic fences or non-fully spanning shock generators. This facilitates the use of the experimental dataset for CFD validation, as done by Bermejo-Moreno et al. (2014). The combination of a simple, low aspect ratio geometry, well-defined boundary conditions, and highly stable freestream conditions means that the flow can be directly replicated by high fidelity simulations. Once such simulations are validated using the converged mean flow and turbulence statistics, the simulation results can be used to further explore the flow physics, including features that are difficult to measure in experiments.

\section{Experimental setup}

\subsection{Facility overview}

Experiments are conducted in the low aspect ratio continuous flow Mach 2.05 wind tunnel at Stanford University. The open-loop wind tunnel sources air from a compressor upstream and exhausts downstream to a muffler and duct to the atmospheric conditions. Moisture, oil, and particulates are removed from the compressed air supply by a refrigerated air dryer, oil separator, and filter. The flow then passes through a regulator which maintains the incoming stagnation pressure at a constant value of $P_{0}=255.3 \pm 1 \mathrm{kPa}$. A chilled water shell and tube heat exchanger and series of electrical resistance heaters allow the stagnation temperature to be maintained at a steady value of $T_{0}=303 \pm 1 \mathrm{~K}$ throughout the experiments. The upstream section of the experiment consists of a $1.54 \mathrm{~m}$ long constant

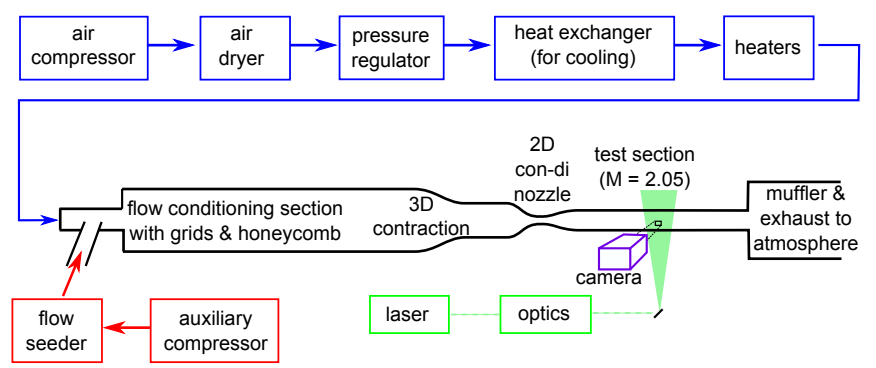

Figure 1: Overview of wind tunnel test facility and PIV setup.

area duct $(127 \mathrm{~mm} \times 114.3 \mathrm{~mm})$ containing two coarse grids, two fine grids, and a honeycomb to condition the flow. The flow then passes through a $3 \mathrm{D}$ contraction and then a $2 \mathrm{D}$ symmetric converging-diverging nozzle which establishes a clean $M_{\infty}=2.05$ inflow to the test section. A schematic overview of the wind tunnel facility is presented in Figure 1.

\subsection{Test section geometries}

For all cases the test section inlet consists of a constant area duct with a cross section of $45 \mathrm{~mm} \times 47.5 \mathrm{~mm}$ which allows the boundary layers to develop over a length of $325 \mathrm{~mm}$ downstream of the exit of the converging-diverging nozzle. This section is followed by a small fully-spanning compression ramp on the top wall which generates an oblique shock wave. Experiments are performed for incident shocks generated by ramps of three different heights: $h_{\text {ramp }}=1.1,3.0$, and $5.0 \mathrm{~mm}$. The $h_{\text {ramp }}=1.1 \mathrm{~mm}$ results were previously presented by Helmer et al. (2012). Selected results for that case will be shown for comparison, but the focus of this paper will be on the new larger ramp geometries. In each case, the ramp is inclined at an angle of $20^{\circ}$ to the incoming flow. All ramps considered are all smaller than the undisturbed incoming boundary layer thickness, $\delta_{0}=5.4 \mathrm{~mm}$, and will henceforth be referred to in nondimensional form as $h_{\text {ramp }} / \delta_{0}=0.20,0.56$, and 0.93 . The three ramp heights will also be referred to as "small", "mid-size", and "large"; however it is important to keep in mind that even the "large" ramp is embedded in the incoming boundary layer.

The subsonic portion of the boundary layer transmits the adverse pressure gradient upstream of the ramp foot and causes the flow to turn before reaching the ramp. When the height of the ramp is small relative to the incoming boundary layer, the angle that the flow must deflect increases with increasing ramp height. This is a very different behavior than the fixed inviscid turning angle required when a large shock generating feature protrudes into the freestream flow. Downstream of the ramp, the top wall of the tunnel turns back to horizontal and the flow continues through a constant area duct with cross section $\left(45-h_{\text {ramp }}\right) \mathrm{mm} \times 47.5 \mathrm{~mm}$ before dumping into a muffler which exhausts to atmospheric conditions. The test section geometry is depicted in Figure 2 and summarized in Table 1.

\subsection{PIV measurements}

Velocity measurements are acquired using high resolution two-component particle image velocimetry (PIV) in streamwise-vertical planes at $z / \delta_{0}=0.74,1.01$, and 3.89, with 
Table 1: Summary of test section geometries

\begin{tabular}{ll}
\hline \hline ramp angle, $\alpha$ & $20^{\circ}$ \\
\hline ramp height, $h_{\text {ramp }}$ & $0.20 \delta_{0}($ small, Helmer $(2011))$ \\
& $0.56 \delta_{0}$ (mid-size) \\
& $0.93 \delta_{0}($ large $)$ \\
\hline cross-section upstream & $45.0 \mathrm{~mm} \times 47.5 \mathrm{~mm}$ \\
\hline cross-section downstream & $\left(45.0-h_{\text {ramp }}\right) \mathrm{mm} \times 47.5 \mathrm{~mm}$ \\
\hline \hline
\end{tabular}

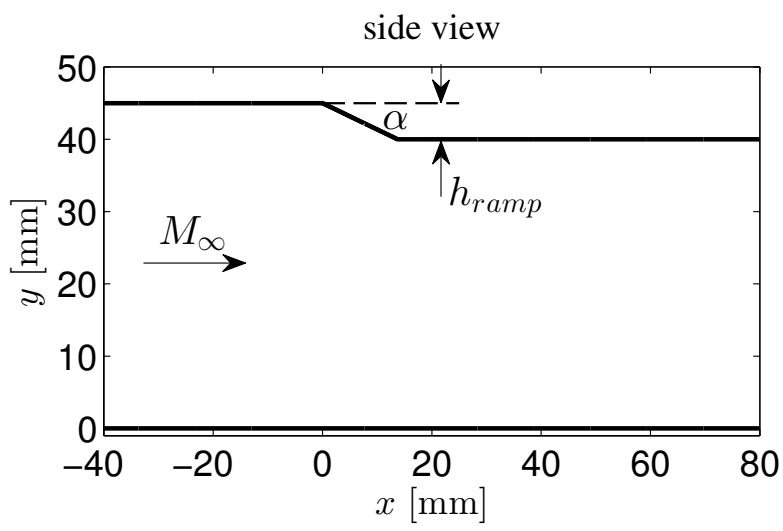

top view

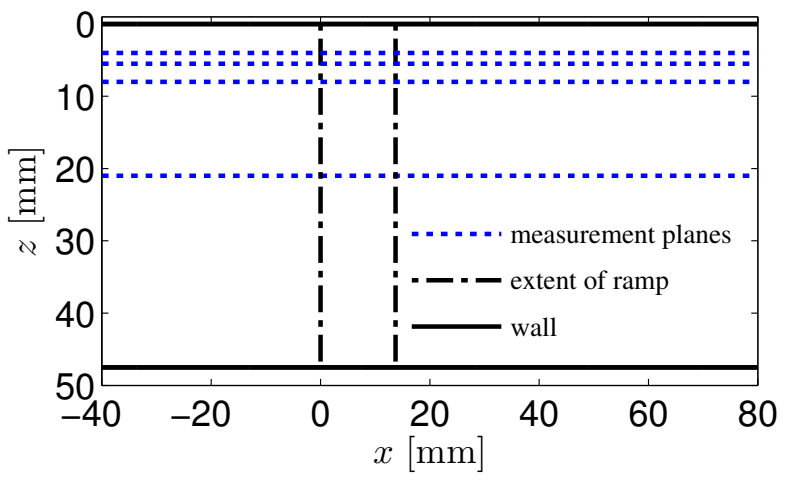

Figure 2: Schematic of test section geometry for the case with the largest compression ramp $\left(h_{\text {ramp }} / \delta_{0}=0.93\right)$. The four streamwise-vertical PIV measurement planes are depicted by dashed blue lines. The inlet cross section $(45 \mathrm{~mm}$ $\times 47.5 \mathrm{~mm})$ and ramp angle $\left(\alpha=20^{\circ}\right)$ are identical for all the ramp heights.

Table 2: Summary of PIV parameters.

\begin{tabular}{ll}
\hline \hline camera aperture & $f / 8$ \\
\hline laser sheet thickness & $0.7 \mathrm{~mm}$ \\
\hline inter-frame time, $\Delta t$ & $0.8 \mu \mathrm{s}$ \\
\hline particle time constant, $\tau_{p}$ & $1.0 \mu \mathrm{s}$ \\
\hline image resolution & $15 \mu \mathrm{m} /$ pixel \\
\hline vector spacing $(50 \%$ overlap) & $0.12 \mathrm{~mm}$ \\
\hline PIV measurement planes $\left[z / \delta_{0}\right]$ & $3.89,1.48,1.01,0.74$ \\
\hline $\begin{array}{l}\text { number of image pairs acquired at } \\
\text { each camera location }\end{array}$ & 5000 \\
\hline \hline
\end{tabular}

the origin of the $z$-coordinate fixed to the back wall of the duct. For the largest ramp geometry $\left(h_{\text {ramp }} / \delta_{0}=0.93\right)$, the threedimensional effects imposed by the side wall interaction extend further outside the side wall boundary layer so an additional PIV measurement plane at $z / \delta_{0}=1.48$ is added. The measurement plane locations are denoted by dashed lines in Figure 2.

Aerosolized olive oil particles are used to seed the flow for imaging. The seed is generated by six Laskin nozzles and introduced upstream of the flow conditioning section to minimize any disturbances to the downstream flow. The particle response through a shock wave was investigated experimentally, giving a particle response time constant of $\tau_{p} \approx 1 \mu \mathrm{s}$. The characteristic time scale of the incoming boundary layer is $\tau=\delta_{0} / U_{\infty} \approx 10 \mu \mathrm{s}$, which leads to a particle Stokes number of $\mathrm{St}=\tau_{p} / \tau \approx 0.1$. A New Wave Solo-200XT dual-pulse PIV laser with a wavelength of $532 \mathrm{~nm}$ is used to generate a light sheet of thickness $0.7 \mathrm{~mm}$. The time between laser pulses is $\Delta t=0.8 \mu$ s for all experiments.

For the cases with $h_{\text {ramp }} / \delta_{0}=0.20$ and 0.56 , PIV image pairs are recorded at $4 \mathrm{~Hz}$ by a TSI model 630047 PIV camera with a $1024 \times 1280$ pixel array and a Nikon AF Micro-Nikkor $200 \mathrm{~mm}$ lens. For the case with the larger compression ramp $\left(h_{\text {ramp }} / \delta_{0}=0.93\right)$, data are acquired at $7 \mathrm{~Hz}$ using a newer TSI PowerPlus 4 MP camera with a $2048 \times 2048$ pixel array and the same lens. In order to achieve a wide-field representation of the flow at high resolution, PIV image pairs are acquired by a single camera at many different locations throughout the test section. Data from each camera position are located in space using an alignment grid with fiducial markings, and a composite representation of the velocity measurements is achieved by stitching all the individual PIV data tiles together. A minimum of 5000 image pairs are recorded at each camera position to give wellconverged mean velocity and turbulence statistics.

\subsection{PIV data processing}

Raw image data are first background-subtracted and then processed using a FFT-based cross-correlation algorithm with an iterative interrogation scheme. The first iteration uses $64 \times 64$ pixel interrogation regions and a spatially varying initial guess for the pixel displacement between interrogation regions. The algorithm then iterates twice at $32 \times 32$ pixel window sizes and does a final pass at $16 \times 16$ pixels with $50 \%$ overlap of vectors. For the $h_{\text {ramp }} / \delta_{0}=0.56$ and 0.93 cases the image resolution is $15 \mu \mathrm{m} / \mathrm{pixel}$, leading to a final vector spacing of $0.12 \mathrm{~mm}$. Helmer et al. acquired data for the smaller ramp case $\left(h_{\text {ramp }} / \delta_{0}=0.20\right)$ at an even higher resolution. In order to achieve a high dynamic range of particle displacements for accurate measurement of both low and high velocity regions, the freestream particle displacement between frames is larger than the size of the final interrogation windows. Therefore in fast regions of the flow, the actual resolution of the velocity vectors in the streamwise direction is dictated by the physical distance traveled by particles between image frames. In the incoming freestream where the mean velocity is $530 \mathrm{~m} / \mathrm{s}$, this distance is approximately $425 \mu \mathrm{m}$. Even considering the effects of particle travel, the measurements presented in this paper are of considerably higher resolution than most previous PIV studies of 


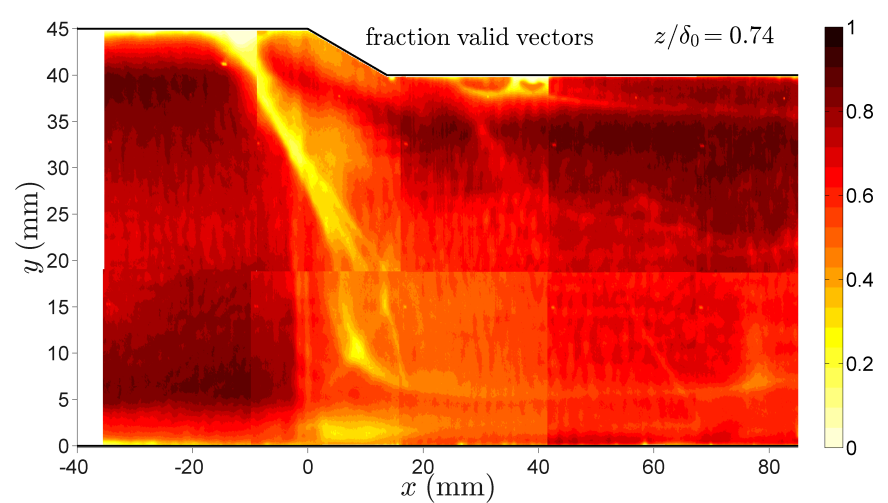

Figure 3: Fraction of valid vectors at each location within the measurement domain for the $z / \delta_{0}=0.74$ plane.

shock boundary layer interaction flows. The trade off of having such high resolution measurements is that the field of view in each camera location is small, meaning that it is impossible to capture instantaneous snapshots of large scale structures in the flow. However, by stitching together the tiles of data, a statistical representation of $U, V, \sqrt{\left\langle u^{\prime} u^{\prime}\right\rangle}, \sqrt{\left\langle v^{\prime} v^{\prime}\right\rangle}$, and $\left\langle u^{\prime} v^{\prime}\right\rangle$ is possible over a large area. The PIV acquisition parameters are summarized in Table 2.

In order to minimize peak-locking, the image magnification and aperture settings are chosen to ensure that the diffractionlimited particle images are resolved $\left(d_{\tau} / d_{\text {pix }}>2\right.$, where $d_{\tau}$ is the particle image diameter and $d_{p i x}$ is the physical size of a pixel). The particle displacements associated with both streamwise and vertical velocity fluctuations are greater than one pixel in most regions of interest in the flow, further mitigating the effects of peak-locking on the turbulence measurements, as described by Christensen (2004).

Instantaneous velocity vector fields are validated using several criteria. First, a correlation coefficient of at least 0.5 is required to consider the cross-correlation peak between interrogation windows valid. Secondly, a range filter is implemented to remove vectors that fall outside a pre-determined span of $U \in[-130,740] \mathrm{m} / \mathrm{s}$ and $V \in[-250,250] \mathrm{m} / \mathrm{s}$. Thirdly, a filter is used to remove outliers, defined as any instantaneous velocity vectors which deviate from the local mean velocity by more than three standard deviations. In regions where spurious correlations with reflections from the walls could occur, the PDFs of instantaneous velocity were carefully examined to ensure the data quality. Finally, data are cropped from the flow field representation in any region in which the yield of valid vectors is less than $10 \%$.

In general, the vector yields are high throughout the flow. This is demonstrated by Figure 3, which shows the fraction of valid vectors in the $z / \delta_{0}=0.74$ data plane for the larger ramp case. This measurement plane presents the largest challenge for reliable data acquisition due to lower seed density within the side wall boundary layer. The small regions of lower valid vector yield are due the large density gradient in the vicinity of the incident shock wave, laser reflections that could not be completely eliminated near the top and bottom walls, and mild

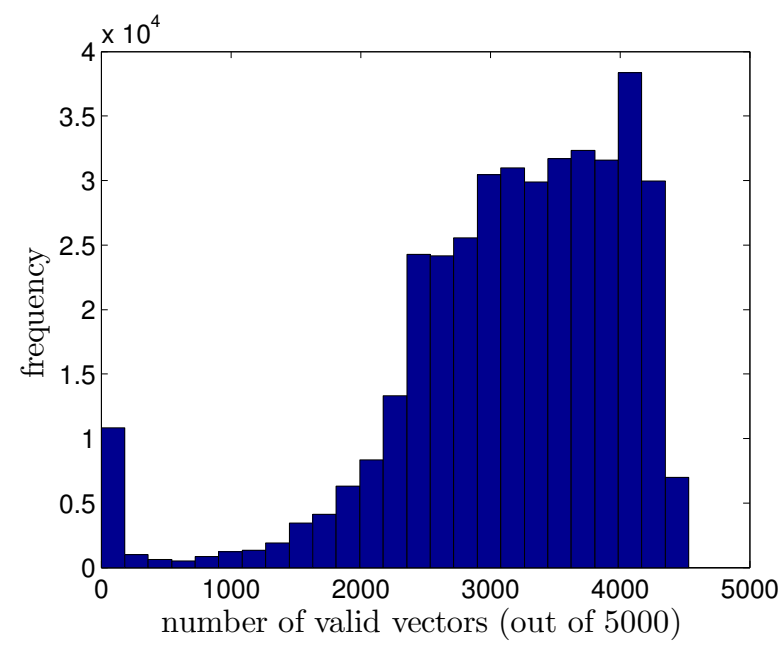

Figure 4: Histogram of valid vector counts (out of 5000) at each data point location in the measurement domain for the $z / \delta_{0}=0.74$ plane.

Table 3: Summary of inlet conditions

\begin{tabular}{ll|ll}
\hline \hline$M_{\infty}$ & $2.05 \pm 0.03$ & $\delta_{0}$ & $5.4 \mathrm{~mm}$ \\
\hline$U_{\infty}$ & $530 \pm 1 \mathrm{~m} / \mathrm{s}$ & $R e_{\delta_{0}}$ & $1.82 \times 10^{5}$ \\
\hline$P_{0}$ & $255.3 \pm 1 \mathrm{kPa}$ & $\theta$ & $0.47 \mathrm{~mm}$ \\
\hline$T_{0}$ & $303 \pm 1 \mathrm{~K}$ & $R e_{\theta}$ & $1.60 \times 10^{4}$ \\
\hline$\dot{m}$ & $0.68 \mathrm{~kg} / \mathrm{s}$ & $U_{\tau}$ & $22.1 \mathrm{~m} / \mathrm{s}$ \\
\hline \hline
\end{tabular}

fouling of the optical window by olive oil seed particles. Figure 4 shows a histogram of the number of valid vectors at each point in the $z / \delta_{0}=0.74$ plane indicating high yields of valid vectors throughout almost all of the measurement domain, even when it is embedded within the side wall boundary layer.

\section{Inflow and boundary conditions}

The inflow conditions were monitored and controlled continuously throughout the experiments to maintain a constant stagnation temperature of $T_{0}=303 \pm 1 \mathrm{~K}$ and stagnation pressure of $P_{0}=255.3 \pm 1 \mathrm{kPa}$. The incoming freestream flow has a streamwise velocity of $U_{\infty}=530 \pm 1 \mathrm{~m} / \mathrm{s}$ and a Mach number of $M_{\infty}=2.05 \pm 0.03$.

Detailed measurements of the inflow conditions were made in order to provide as much information as possible on boundary conditions for CFD simulations. To avoid confusion in the following discussion, the boundary layers growing on the top and bottom walls will be referred to as vertical boundary layers, while the boundary layer on the side wall will be called the side wall boundary layer. Profiles of the mean and fluctuating velocity components in the undisturbed incoming vertical boundary layer were extracted from PIV measurements on the upper surface at a station $35 \mathrm{~mm}$ upstream of the foot of the shock-generating ramp. These profiles are shown in Figures 5 and 6. Note that only every third data point is plotted to make the figures more readable. 


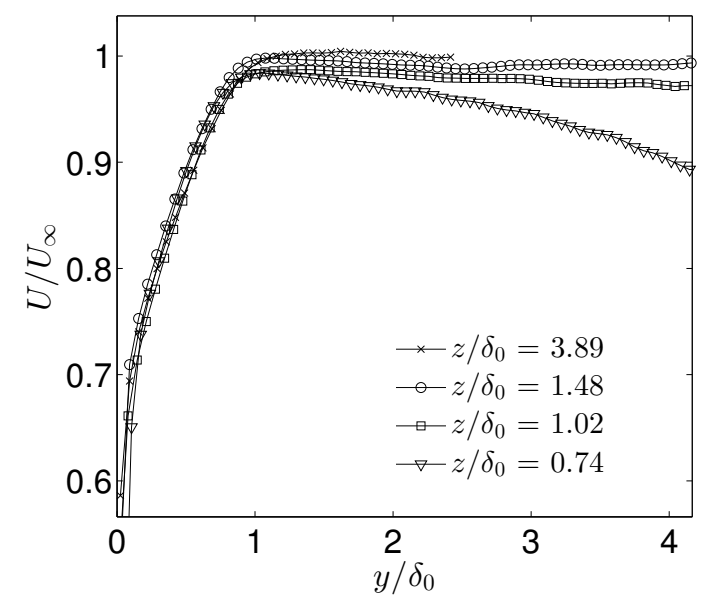

Figure 5: Profiles of mean streamwise velocity at four stations across the tunnel span. Profiles are taken at a fixed streamwise position of $x=-35 \mathrm{~mm}$ $\left(x / \delta_{0}=-6.48\right)$, which is upstream of any influence of the compression ramp. The vertical $(y)$ coordinate extends to the vertical half-height of the tunnel.

The undisturbed incoming vertical boundary layer has $99 \%$ velocity thickness $\delta_{0}=5.4 \mathrm{~mm}$, and momentum thickness $\theta=$ $0.47 \mathrm{~mm}$. The appropriate Reynolds numbers associated with the incoming boundary layer are $R e_{\delta_{0}}=1.82 \times 10^{5}$ and $R e_{\theta}=$ $1.60 \times 10^{4}$, based on the freestream viscosity and density. These inlet conditions are summarized in Table 3.

Profiles of mean streamwise velocity, streamwise fluctuations, vertical fluctuations, and Reynolds shear stress at the inlet to the measurement domain are plotted in Figures 5 and 6. The turbulence quantities are not density-weighted $\left(\sqrt{\rho / \rho_{w}}\right)$ because accurate computation of the density for the profiles inside the side wall boundary layer is not possible. Positive vertical velocities are defined as away from the top wall. Measurements extend across the full height of the duct at the inlet station, with boundary layers on both top and bottom walls resolved. The mean velocity and normal stresses are found to be symmetric about the vertical half-height of the duct, while the shear stresses are anti-symmetric. This symmetry is demonstrated by the profiles of $\sqrt{\left\langle u^{\prime} u^{\prime}\right\rangle} / U_{\infty}$ in Figure 6 a which are plotted across the full height of the duct. The other profiles are plotted to the duct half height $\left(y / \delta_{0}=4.17\right)$ to provide better detail in the vertical boundary layer.

The mean streamwise velocity profiles at different spanwise locations in Figure 5 collapse within the vertical boundary layer, $y / \delta_{0} \leq 1$. This suggests that the wall shear stress does not vary significantly across the span in the undisturbed inlet section. However, outside of $y / \delta_{0}=1$ the profiles deviate significantly. The profile inside the side wall boundary layer $\left(z / \delta_{0}=0.74\right)$ shows a distinct non-monotonic shape, with a maximum velocity at the edge of the vertical boundary layer $\left(y / \delta_{0}=1\right)$ and a decay to $\approx 0.9 U_{\infty}$ at the half-height of the duct. This overshoot effect is also present in the velocity profile at $z / \delta_{0}=1.02$, but it is far less pronounced. The velocity profile at $z / \delta_{0}=1.48$ agrees very closely with the near-centerline profile $\left(z / \delta_{0}=3.89\right)$; the only difference is a very slightly $(\approx 1 \%)$ lower freestream asymptotic velocity. The mean vertical ve- locity everywhere in the inlet section deviates from zero by no more than $+4 \%$ and $-2 \%$ of $U_{\infty}$, and is within $\pm 1 \%$ of $U_{\infty}$ in most regions of the inlet.

Profiles of the streamwise and vertical velocity fluctuations at four locations across the span are shown in Figures $6 \mathrm{a}$ and $6 \mathrm{~b}$, respectively. Similar to the behavior of the mean streamwise velocity, the profiles of velocity fluctuations collapse within the vertical boundary layer, $y / \delta_{0} \leq 1$, and deviate outside this region. For both the streamwise and vertical fluctuations, the profile inside the side wall boundary layer $\left(z / \delta_{0}=0.74\right)$ exhibits a minimum near the edge of the vertical boundary layer and then a gradual increase until the duct half-height $\left(y / \delta_{0}=4.17\right)$. The effect is present, but less pronounced, for the profiles at the edge of the side wall boundary layer $\left(z / \delta_{0}=1.02\right)$. Near the centerline $\left(z / \delta_{0}=3.89\right)$ and outside the vertical boundary layer $\left(y / \delta_{0}>1\right)$, the magnitude of the streamwise and vertical fluctuations decay to $<1 \%$ of $U_{\infty}$. This measurement represents both the freestream turbulence level as well as any overall unsteadiness, and therefore indicates that the inlet flow is clean and does not exhibit any significant unsteadiness. Unlike the mean velocity and normal stresses, the shear stress profiles (Figure 6c) collapse everywhere. The only deviation is a slightly lower peak in $-\left\langle u^{\prime} v^{\prime}\right\rangle$ near the centerline $\left(z / \delta_{0}=3.89\right)$.

These profiles of the flow upstream of the interaction show that the flow is clearly three-dimensional in the inlet section. The non-monotonic velocity profiles at the edge and inside the side wall boundary layer are consistent with the presence of corner vortices as expected in a supersonic duct flow (see e.g. Davis et al. (1986) or Davis and Gessner (1989)). The experimental configuration does not allow for direct measurements of the velocity in cross planes as would be required to fully document the corner vortices upstream of the SBLI. BermejoMoreno et al. (2014) performed a wall-modeled large eddy simulation of the test cases presented in this paper. They computed the upstream flow through the square duct and matched profiles to the experimental inlet data provided at $x=-35 \mathrm{~mm}$. Their fully three-dimensional results show corner vortices with peak cross flow velocity magnitudes of around $1.5 \%$ of the centerline velocity at $x=-35 \mathrm{~mm}$. Profiles of $U, V, u^{\prime}, v^{\prime}$, and $u^{\prime} v^{\prime}$ extracted from the LES show good agreement with the experimental inlet profiles shown in Figures 5 and 6. Based on our collaborations with Bermejo-Moreno et al. (2014), we believe that correct representation of these secondary flow features in the inlet section is critical for accurate CFD prediction of the rest of the flowfield.

The incoming side wall boundary layers are expected to be very similar to the top wall boundary layer where the inlet profile measurements are made. While direct measurements of the side wall boundary layers are not available, this assumption can be justified using the following reasoning. The inlet contraction used to accelerate the flow to $M=1$ is designed using a fifth order polynomial with zero curvature at both endpoints. Furthermore the nozzle length to contraction ratio is $L / H=10$, indicating gentle and continuous curvature throughout. This design is known to minimize secondary flows due to transverse pressure gradients caused by streamline curvature. Any differences in the boundary layers at the exit of the converging-diverging 


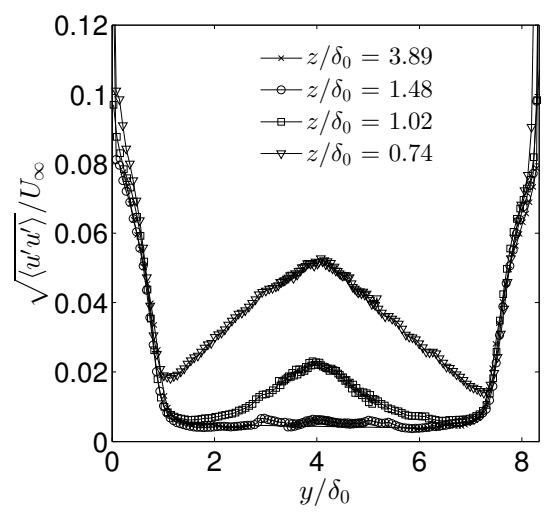

(a)

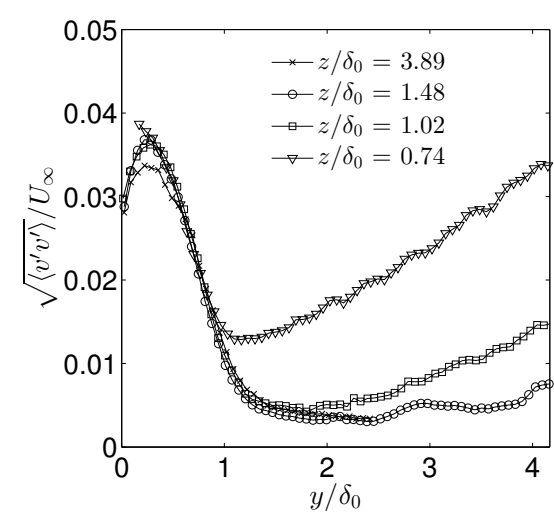

(b)

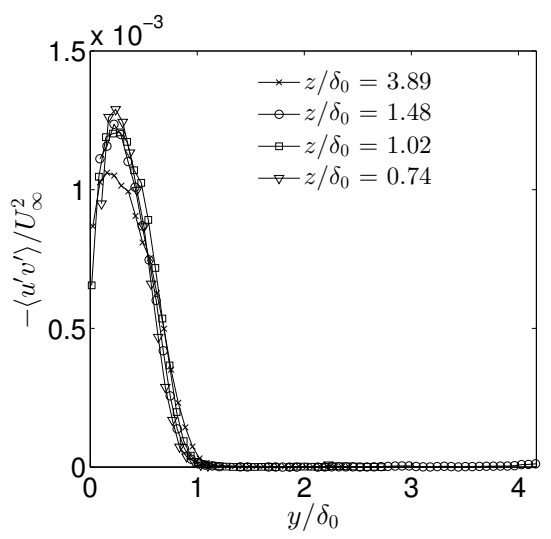

(c)

Figure 6: Profiles of (a) streamwise velocity fluctuations; (b) vertical velocity fluctuations; (c) Reynolds shear stresses; at four stations across the tunnel span. Profiles are taken at a fixed streamwise position of $x=-35 \mathrm{~mm}\left(x / \delta_{0}=-6.48\right)$, which is upstream of any influence of the compression ramp. The vertical (y) coordinate extends the full height of the duct in plot (a) and to the vertical half-height of the tunnel in plots (b) and (c).

nozzle are minimal because all four boundary layers are extremely thin due to the large favorable pressure gradient. The boundary layers on all walls become fully turbulent a short distance downstream $(\approx 6 \mathrm{~mm})$ and develop similarly through the approximately zero pressure gradient square duct upstream of the measurement domain.

The wind tunnel is allowed to run continuously for at least 20 minutes prior to any data acquisition, allowing the whole system to achieve a thermal steady state. For all experiments, the adiabatic wall temperature in the test section inlet is $T_{a w}=$ $288 \mathrm{~K}$, based on a recovery factor of $r=0.89$ and stagnation temperature of $T_{0}=303 \mathrm{~K}$. This temperature is very close to the ambient temperature, and therefore the small heat flux due to natural convection on the outer surfaces of the wind tunnel may be considered negligible. For CFD simulations of this experimental configuration, adiabatic wall boundary conditions are recommended.

\section{Results and discussion}

\subsection{Overview of shock and interaction features}

Figures 7 and 8 depict the primary flow features near the spanwise centerplane of the duct for the $h_{\text {ramp }} / \delta_{0}=0.56$ and $h_{\text {ramp }} / \delta_{0}=0.93$ test section geometries, respectively. In both cases, the incident shock wave (IS) is generated by the $20^{\circ}$ compression ramp on the top wall. An expansion fan is generated at the downstream end of the ramp where the top wall turns back to horizontal. In the smaller ramp case, there is a regular reflection of the shock wave off the bottom wall. This is quite different than for the larger ramp geometry, which involves a Mach reflection. In both cases, compression waves emanate from the bottom wall upstream of the projected impingement point of the incident shock, and these waves coalesce into the separation shock (SS). In the case of the larger ramp, the Mach stem (MS) sits near the core of the duct, resulting in a large region of subsonic flow downstream, as indicated by the approximate sonic line in Figure 8. The subsonic flow caused by the Mach stem is re-accelerated downstream by the expansion fans emanating from the end of the compression ramp and from the separation bubble. In both cases, the transmitted shock (TS) extends down toward the bottom wall until the point where the boundary layer becomes subsonic. The reflected shock (RS) departs from the intersection of the incident shock and Mach stem in the case of the Mach reflection, and from the intersection of the incident and separation shocks for the case of the regular reflection. In the larger ramp geometry, a second reflection off of the top wall (RS2) occurs and this shock interacts with the wake downstream of the Mach stem; however these features are not the primary focus of this investigation.

It is also interesting to note that the shock angles vary with the size of the compression ramp and that for a given geometry there is a high degree of non-uniformity in the shock angles across the span. The incident and reflected shock angles for both test geometries are reported in Table 4 and compared to the results of Helmer (2011) and Helmer et al. (2012), who investigated the flow field for the smaller ramp geometry $\left(h_{\text {ramp }} / \delta_{0}=\right.$ 0.20) with all of the other test parameters matching the current experiments. In some cases, the shock waves are curved so a range of angles are reported. Curvature of the incident shock for the $h_{\text {ramp }} / \delta_{0}=0.56$ case is also noted in the wall-modeled LES of the same configuration performed by Bermejo-Moreno et al. (2014). We are confident that this effect is physical and believe that it is related to the strong three-dimensional nature of this flow, but unfortunately do not have a definitive explanation for it. It should be noted that in both the $h_{\text {ramp }} / \delta_{0}=0.56$ and 0.93 cases, the incident shock angle near the centerline is approximately the same near the top of the domain. In the midsize ramp case, the shock steepens as it approaches the bottom wall. In the larger ramp case, this steepening of the incident shock wave is not observed; however the incident shock wave terminates in the core of the flow at the top of the Mach stem.

For all of the test cases, the shock angles steepen as the side wall is approached. This is consistent with the fact that the 
Table 4: Incident and reflected shock angles observed in each of the PIV measurement planes for both of the ramp geometries considered in the present experiments $\left(h_{\text {ramp }} / \delta_{0}=0.56\right.$ and 0.93$)$, compared with the results of Helmer et al. $\left(h_{\text {ramp }} / \delta_{0}=0.20\right)$.

\begin{tabular}{lllll}
\hline \hline & $z / \delta_{0}=3.89$ & $z / \delta_{0}=1.42$ & $z / \delta_{0}=1.02$ & $z / \delta_{0}=0.74$ \\
\hline \hline IS $\left(h_{\text {ramp }} / \delta_{0}=0.93\right)$ & $-37^{\circ}$ & $-41^{\circ}$ & $-45^{\circ}$ & $-47^{\circ}$ \\
\hline IS $\left(h_{\text {ramp }} / \delta_{0}=0.56\right)$ & $-38^{\circ}$ to $-44^{\circ}$ & no data & $-39^{\circ}$ to $-47^{\circ}$ & $-40^{\circ}$ to $-48^{\circ}$ \\
\hline IS $\left(h_{\text {ramp }} / \delta_{0}=0.20\right)$ & $-37^{\circ}$ & no data & $-39^{\circ}$ & $-40^{\circ}$ \\
\hline $\operatorname{RS}\left(h_{\text {ramp }} / \delta_{0}=0.93\right)$ & $54^{\circ}$ to $44^{\circ}$ & $47^{\circ}$ & $47^{\circ}$ & can't be identified \\
\hline $\operatorname{RS}\left(h_{\text {ramp }} / \delta_{0}=0.56\right)$ & $44^{\circ}$ & no data & $46^{\circ}$ & $48^{\circ}$ \\
\hline $\operatorname{RS}\left(h_{\text {ramp }} / \delta_{0}=0.20\right)$ & $38^{\circ}$ & no data & $38^{\circ}$ & $40^{\circ}$ \\
\hline \hline
\end{tabular}

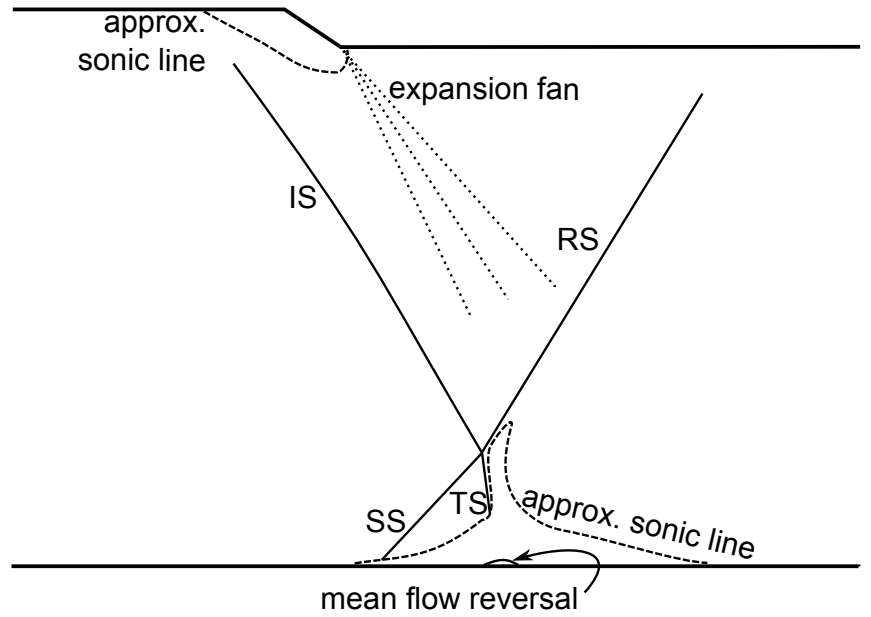

Figure 7: Shock and interaction features near the spanwise centerplane of the duct for the $h_{\text {ramp }} / \delta_{0}=0.56$ geometry. This figure depicts a regular reflection of the incident shock wave.

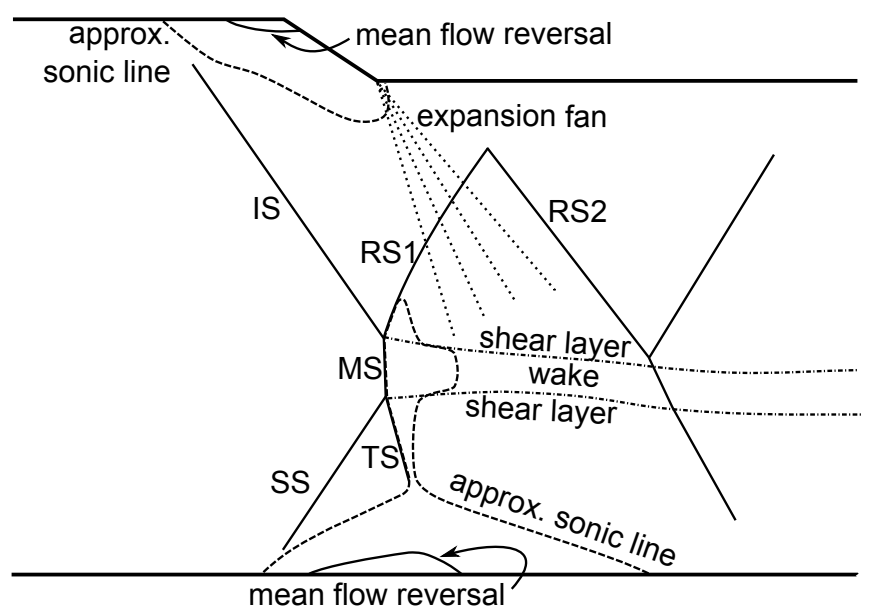

Figure 8: Shock and interaction features near the spanwise centerplane of the duct for the $h_{\text {ramp }} / \delta_{0}=0.93$ geometry. This figure depicts a Mach reflection of the incident shock wave. incoming flow is slower near and inside the side wall boundary layer. The steepening shock angle could also be related to oblique shock waves emanating from the side wall; however without data in streamwise-spanwise planes this is difficult to confirm. In all cases, the angle of the incident shock wave is considerably lower than the $-52^{\circ}$ angle predicted by inviscid theory for a Mach 2 flow impinging on a $20^{\circ}$ ramp. This indicates that the small ramp height relative to the incoming boundary layer, viscous effects near the foot of the ramp, and confinement effects due to the side walls play an important role in determining the freestream behavior of the incident shock wave.

In the case of the largest ramp geometry, the angles of the separation and transmitted shocks can also be identified in the $z / \delta_{0}=3.89$ and 1.48 planes. In the planes closer to the sidewall, these features are no longer identifiable due to the high levels of velocity fluctuations associated with the thickening of the side wall boundary layer. Near the centerplane $\left(z / \delta_{0}=3.89\right)$, the separation and transmitted shocks have angles $38^{\circ}$ and $-60^{\circ}$, respectively. Off center, but outside the side wall boundary layer $\left(z / \delta_{0}=1.48\right)$, the separation and transmitted shock angles are $43^{\circ}$ and $-76^{\circ}$, respectively. These results are consistent with the trend of steeper shocks away from the centerline that was noted in the incident and reflected shock angles in Table 4.

\subsection{Mean velocity field comparisons}

Mean streamwise velocity contours for the flow in the midsize and large ramp test geometries are shown in Figures 9 and 10 , respectively. Note that the $x$ and $y$ axes are scaled differently. Blank areas in the plots correspond to regions where data were not acquired or where data were deemed invalid based on the criteria described in section 2.3. In the mid-size ramp case (Figure 9) the interaction regions are largely confined to the vicinity of the compression ramp and the impingement point of the incident shock, so measurements are acquired selectively in these regions. The SBLI caused by the larger ramp geometry (Figure 10) is considerably stronger and exhibits a higher degree of three-dimensionality. For this reason, measurements are made throughout the entire test section and an additional plane of PIV data is acquired at $z / \delta_{0}=1.48$.

The most prominent difference between the shock structures produced by the mid-size $\left(h_{\text {ramp }} / \delta_{0}=0.56\right)$ and large $\left(h_{\text {ramp }} / \delta_{0}=0.93\right)$ ramp cases is the presence of a Mach stem for the larger ramp geometry, as described in section 4.1. This feature is evident in the mean velocity data near the center of 

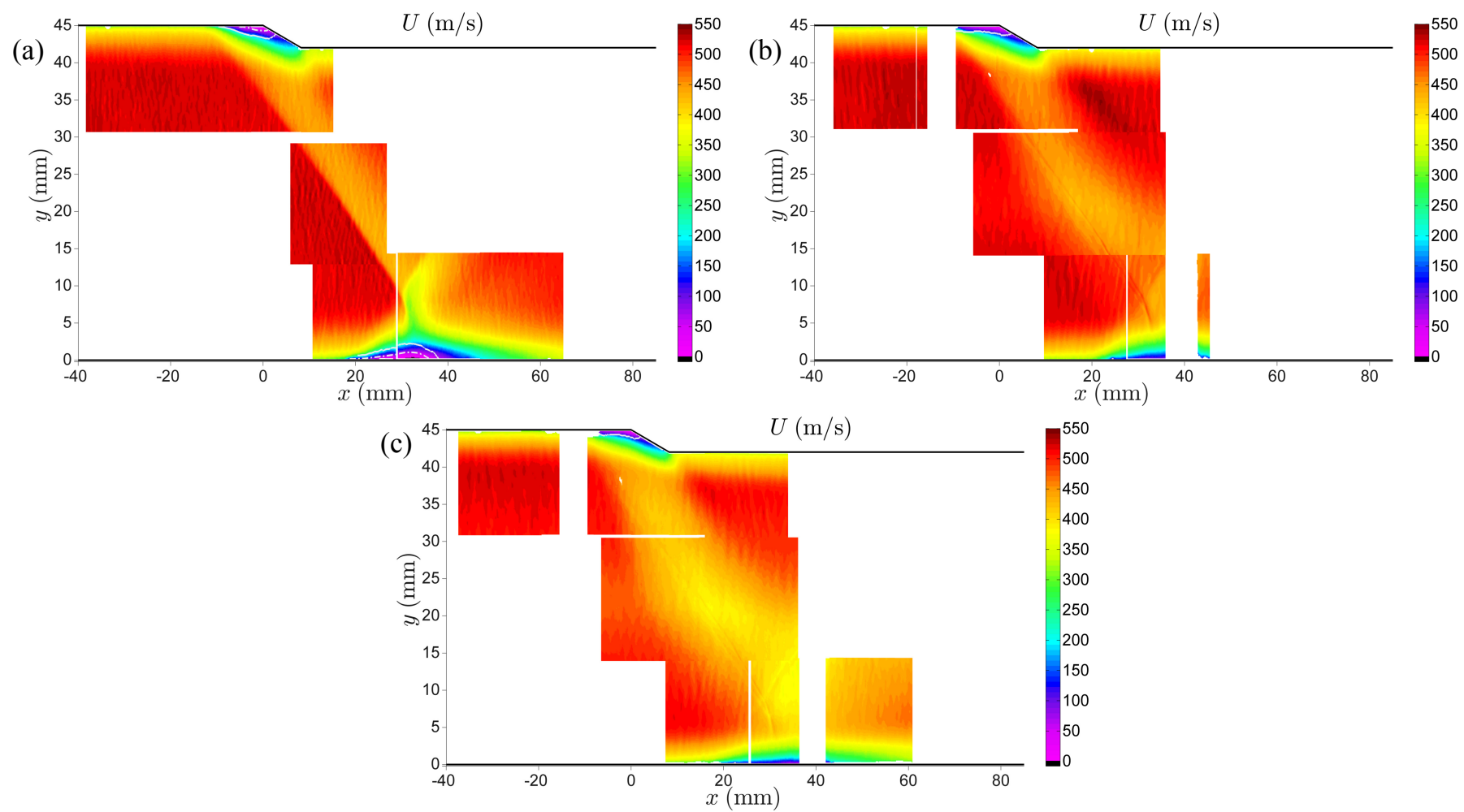

Figure 9: Mean streamwise velocity for the mid-size ramp case $\left(h_{\text {ramp }} / \delta_{0}=0.56\right)$ in three planes across the tunnel span. $(\mathrm{a}) z / \delta_{0}=3.89($ near centerline $),(\mathrm{b})$ $z / \delta_{0}=1.02$, and (c) $z / \delta_{0}=0.74$. Reversed flow probability isocontours ( $\longrightarrow 5 \%$ and - - $\cdot 25 \%$ ) are superimposed.
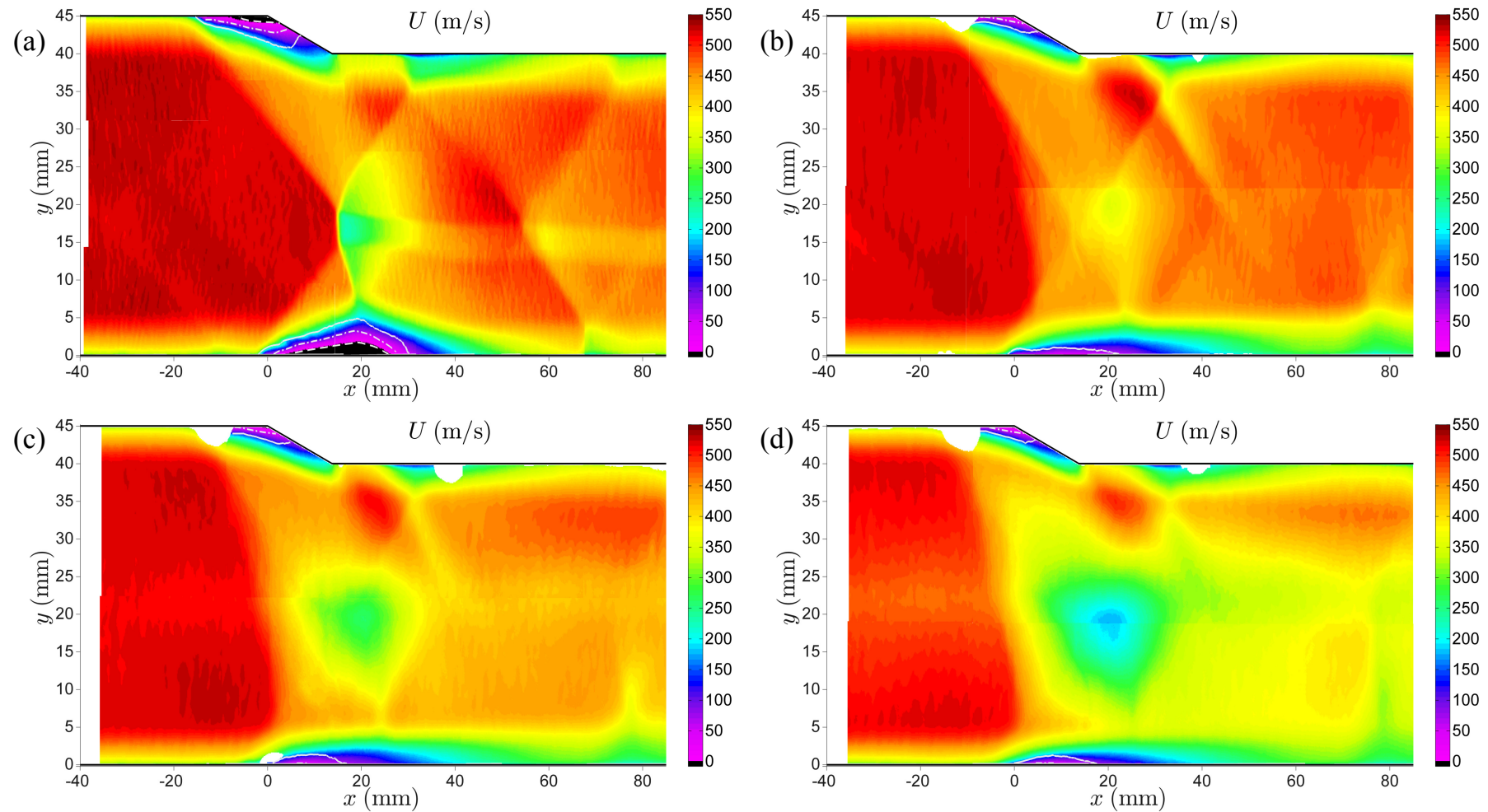

Figure 10: Mean streamwise velocity for the largest ramp case $\left(h_{\text {ramp }} / \delta_{0}=0.93\right)$ in four planes across the tunnel span. $(\mathrm{a}) z / \delta_{0}=3.89$ (near centerline), $(\mathrm{b})$

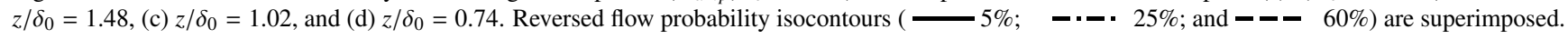



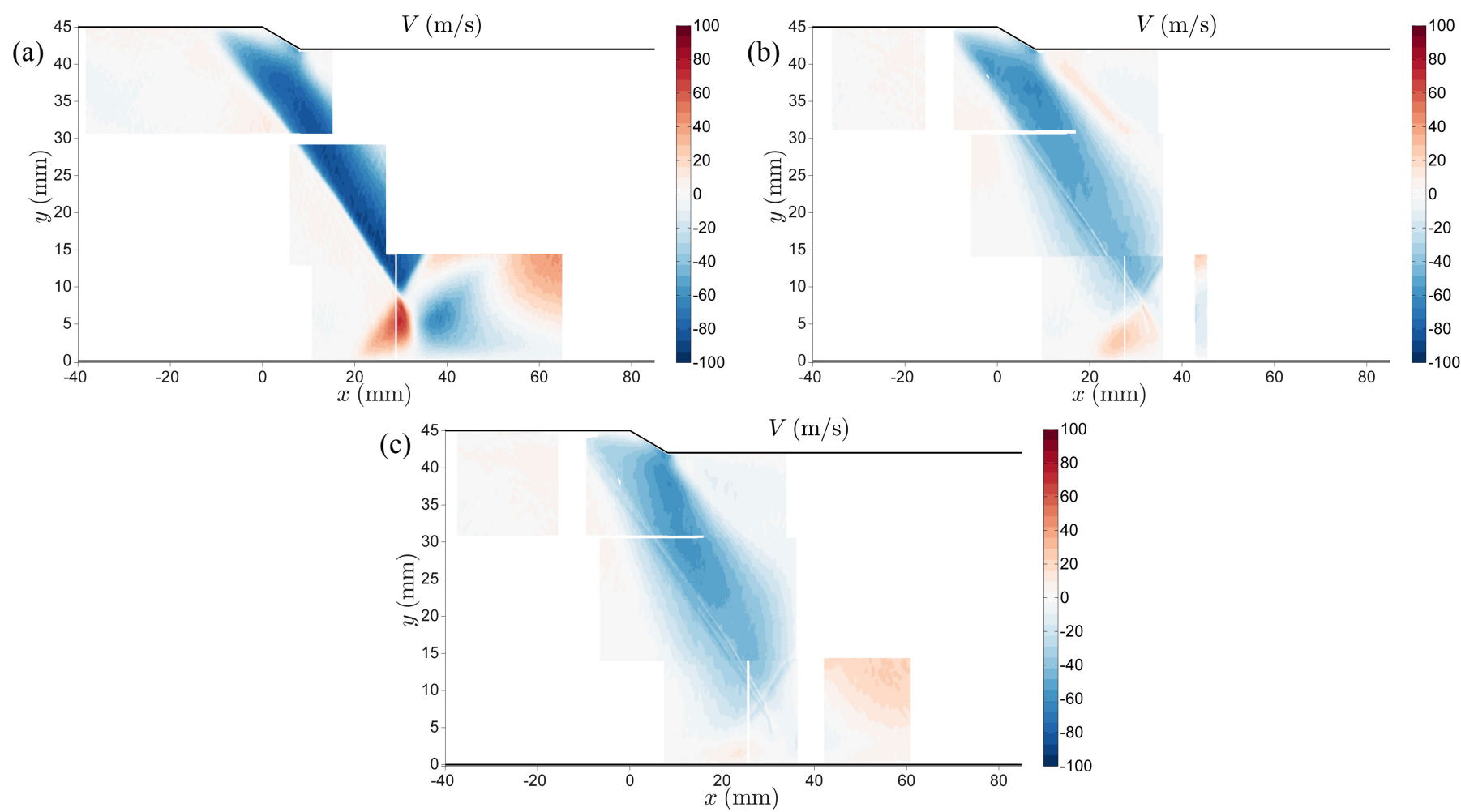

Figure 11: Mean vertical velocity for the mid-size ramp case $\left(h_{\text {ramp }} / \delta_{0}=0.56\right)$ in three planes across the tunnel span. $(a) z / \delta_{0}=3.89($ near centerline), $(b)$ $z / \delta_{0}=1.02$, and (c) $z / \delta_{0}=0.74$.
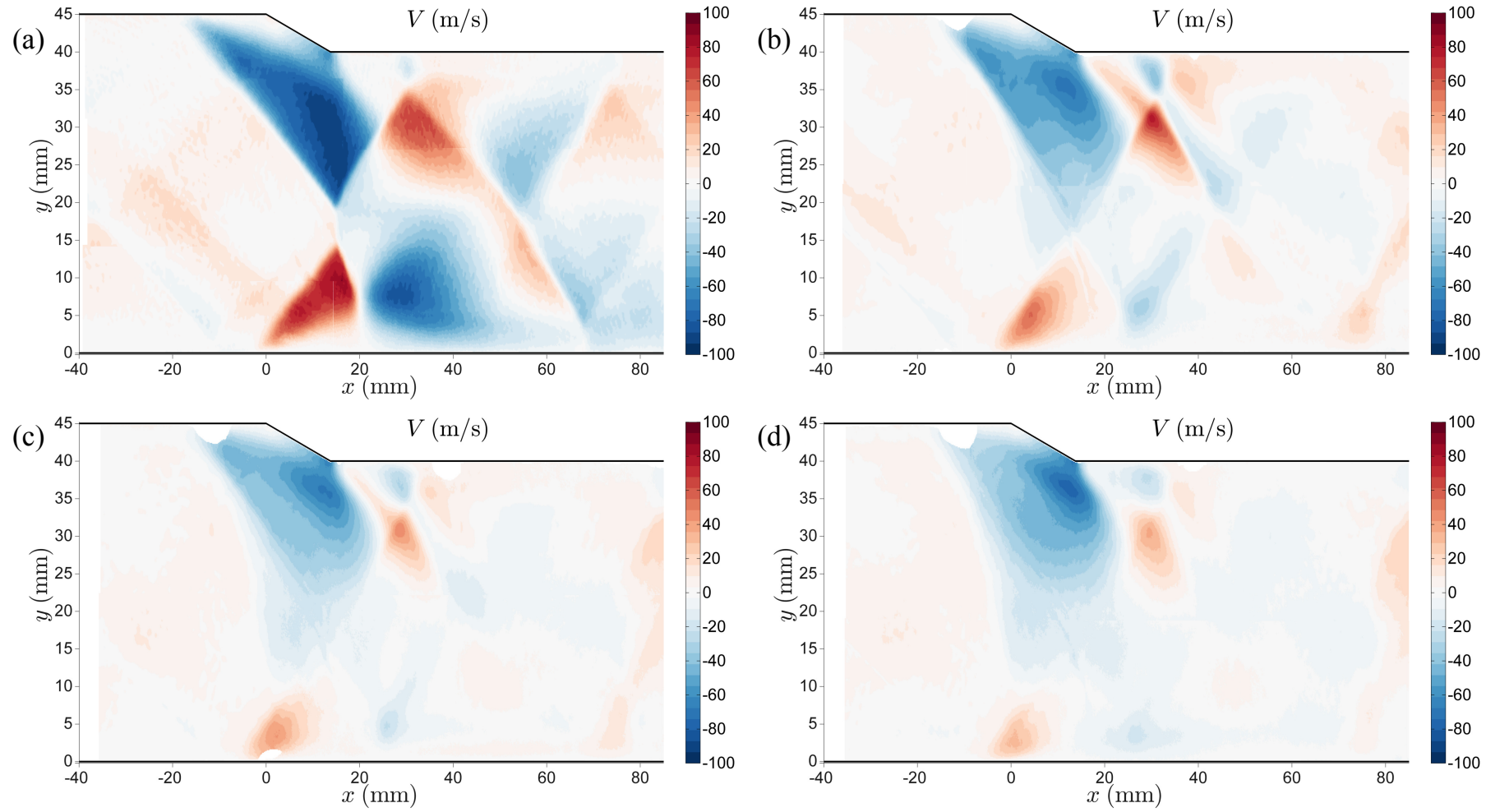

Figure 12: Mean vertical velocity for the largest ramp case $\left(h_{\text {ramp }} / \delta_{0}=0.93\right)$ in four planes across the tunnel span. $(\mathrm{a}) z / \delta_{0}=3.89($ near centerline $),(\mathrm{b}) z / \delta_{0}=1.48$, (c) $z / \delta_{0}=1.02$, and (d) $z / \delta_{0}=0.74$. 
the span $\left(z / \delta_{0}=3.89\right)$, but it vanishes closer to the side wall. Even though the $z / \delta_{0}=1.48$ plane resides outside of the undisturbed upstream sidewall boundary layer, the shock wave pattern and interaction zone in this plane are clearly affected by the interaction of the shocks with the side wall boundary layer. As the side wall is approached, the streamwise deceleration of the flow begins further upstream and is more gradual than the sharp deceleration across the incident shock wave in the core of the flow. In addition, subsonic flow is observed near the vertical centerline for the $z / \delta_{0}=1.02$ and 0.74 planes. This is not due to the presence of a Mach stem in these planes, but rather due to the thickening of the side wall boundary layer as it interacts with the shock waves. These results are qualitatively consistent with the observations of Hanada et al. (2005). That group found a high degree of spanwise non-uniformity in a normal shock boundary layer interaction in a duct, with a large low velocity region in the core of the flow and higher velocities off-center.

The transition from regular reflection to Mach reflection has been well studied for shock-shock interactions generated by two symmetric wedges (see e.g. Hornung and Robinson (1982), Skews (2000), or Ivanov et al. (2001)), but fewer studies have been done where the Mach reflection is part of an incident/reflected SBLI. Tieng et al. (1992) studied this phenomenon using holographic interferography for a number of test cases in which both the incoming Mach number and deflection angle across the incident shock were altered. Matheis et al. (2013) studied the transition from regular reflection to Mach reflection in a Mach 2 incident shock boundary layer interaction using large eddy simulation. They present spanwise-averaged results which show that the nominal transition from regular reflection to Mach reflection occurred when a flow deflection of $13^{\circ}$ was achieved across the incident shock wave. BermejoMoreno et al. (2014) performed two wall-modeled large eddy simulations of the $h_{\text {ramp }} / \delta_{0}=0.93$ geometry presented in this paper - one treating the side walls as no-slip surfaces and one using spanwise periodic boundary conditions for the side walls. They found that for the case with no-slip side walls, a Mach stem is predicted; however for the same geometry but with spanwise periodic boundary conditions, a regular shock reflection is predicted. This indicates that the effects of confinement by the side wall boundary layers is critical in accurately predicting the flow throughout the domain.

In the SBLI on the top wall near the foot of the compression ramp, no mean flow reversal is observed anywhere across the span for the mid-size ramp geometry $\left(h_{\text {ramp }} / \delta_{0}=0.56\right)$. However the flow is intermittently reversed, with probabilities of reversed flow up to $\approx 53 \%$ at the foot of the compression ramp near the spanwise centerline of the duct. Closer to the side wall $\left(z / \delta_{0}=1.02\right.$ and 0.74$)$, the region of intermittent flow reversal does not extend vertically as far from the top wall and the maximum probability of observing reversed flow is reduced to $\approx 45 \%$. The stronger compression ramp SBLI produced by the larger ramp $\left(h_{\text {ramp }} / \delta_{0}=0.93\right)$ involves a small region of mean flow reversal at the foot of the compression ramp near the spanwise centerline, denoted by the black contour in Figure 10a. Although the flow is reversed on average, the maximum probability of instantaneously reversed flow observed in this re- gion is only $\approx 79 \%$. Away from the spanwise centerline of the duct, the region of intermittent flow reversal decreases in size, as can be seen by the isocontours of reversed flow probability superimposed on the mean streamwise velocity plots in Figures 10a-d. Furthermore, the maximum probability of reversed flow observed at the foot of the $h_{\text {ramp }} / \delta_{0}=0.93$ compression ramp drops to $\approx 58 \%, 55 \%$, and $52 \%$ at $z / \delta_{0}=1.48,1.02$, and 0.74 , respectively.

In the SBLI caused by the incident shock impinging on the bottom wall boundary layer, a small region of mean flow reversal is observed near the centerline in the $h_{\text {ramp }} / \delta_{0}=0.56$ ramp geometry. A significantly larger region of mean flow reversal occurs in the stronger incident SBLI caused by the larger $\left(h_{\text {ramp }} / \delta_{0}=0.93\right)$ ramp configuration. These regions are denoted by the black contours near the bottom wall in Figures 9a and 10a. To give an idea of the sizes of the separated zone, isocontours of reversed flow probability at levels of 5\%, 25\%, and $60 \%$ are superimposed on top of the mean streamwise velocity plots. No mean flow reversal is observed in any of the off-center planes; however there are still significant regions of intermittently reversed flow. In the work of Helmer (2011), no mean flow reversal was observed anywhere in the SBLIs generated by the $h_{\text {ramp }} / \delta_{0}=0.20$ case, and the maximum observed probability of flow reversal was $\approx 20 \%$, which is significantly lower than the levels observed in the current experimental geometries.

In both the mid-size and large ramp geometries, the bottom wall boundary layer thickens rapidly due to the adverse pressure gradient imposed by the incident shock wave. This thickening is most severe near the centerline of the tunnel for both geometries. This effect is partially due to the non-uniform shock strength across the span of the tunnel. In addition, the region of mean flow reversal on the bottom wall causes a large blockage near the center of the duct, forcing higher momentum fluid to divert upward and toward the side walls. This energizes the vertical boundary layer at locations away from the spanwise centerplane of the duct, mitigating the thickening due to the shock interaction. This variation across the span is significantly stronger in the interaction caused by the large ramp geometry.

Figure 10 shows that the variation in the boundary layer thickening across the height of the duct on the side wall is qualitatively similar to the variation of bottom wall boundary layer separation and thickening. The flow is very slow and the boundary layer is highly distorted in the region at the vertical centerline and close to the side wall $(x \approx 20 \mathrm{~mm}, y \approx 25 \mathrm{~mm}$, $z=4 \mathrm{~mm}$ ). Below this low velocity region but above the bottom wall boundary layer, the flow is significantly faster. Above the region where the side wall boundary layer is thickest, there is another region of faster flow due to acceleration through the expansion fan. These features at $z / \delta_{0}=0.74$ indicate that the side wall boundary layer distortion is greatest near the vertical centerline and that higher momentum air is diverted around that blockage, in the same manner as previously described for the bottom wall boundary layer.

Another interesting characteristic of the incident shock interaction for the larger ramp geometry is that near the spanwise centerline $\left(z / \delta_{0}=3.89\right)$, the vertical boundary thins rapidly 
downstream of the transmitted shock. However, this vertical boundary layer thinning occurs much more gradually in the offcenter planes $\left(z / \delta_{0}=1.48,1.02,0.74\right)$. Another way to look at this effect is to examine the mean vertical velocity contours in Figure 12. The incident shock interaction involves a region of strong positive vertical velocity due to the separation shock, followed downstream by a region strong negative velocity caused by the transmitted shock and the expansion fan emanating from the point where it impinges on the sonic line. The vertical velocities are strongest near the spanwise centerline, and the magnitudes of the positive and negative velocities are comparable (Figure 12a). Off the spanwise centerline, the vertical velocities are weaker overall, and the positive vertical velocity region pushing flow away from the wall dominates over the negative vertical velocities driving the recovery (Figures $12 \mathrm{~b}-\mathrm{d}$ ). This is due to a mismatch in the strengths of the separation and transmitted shocks at these off-center locations. The vertical boundary layer on the bottom wall does not recover fully before the next shock impingement at $x \approx 70 \mathrm{~mm}$. The overall degree of vertical boundary layer thickening and thinning is less severe in the mid size ramp case (Figures 9 and 11), and the discrepancies between the center and off-center planes appear less drastic.

\subsection{Turbulence quantities}

Contour plots of the normalized streamwise velocity fluctuations, $\sqrt{\left\langle u^{\prime} u^{\prime}\right\rangle} / U_{\infty}$, in each of the PIV measurement planes for the $h_{\text {ramp }} / \delta_{0}=0.56$ and 0.93 geometries are shown in Figures 13 and 14, respectively. The normalized vertical velocity fluctuations, $\sqrt{\left\langle v^{\prime} v^{\prime}\right\rangle} / U_{\infty}$, appear in Figures 15 and 16, and the Reynolds shear stresses, $\left\langle u^{\prime} v^{\prime}\right\rangle / U_{\infty}^{2}$ are shown in Figures 17 and 18. Because $\sqrt{\left\langle u^{\prime} u^{\prime}\right\rangle}>\sqrt{\left\langle v^{\prime} v^{\prime}\right\rangle}$ throughout most of the flow field, the range of values in the colorbar is scaled down by a factor of two for the vertical fluctuations.

The shock features are clearly distinguishable in all of the fluctuating quantities. In particular, the oblique shock waves are most easily identified in the vertical fluctuations and the nearly normal Mach stem is most apparent in the streamwise velocity fluctuations. This is because of the steep gradient in velocity across the shock waves, such that any slight unsteadiness in the shock feature position appears as an artificially inflated level of turbulence intensity. This unsteadiness is not the focus of the current investigation; however the length scales associated with any motion of the shock waves are discussed later in section 4.5. The positions of the shock features are clearest near the spanwise centerplane $\left(z / \delta_{0}=3.89\right)$ due to the very low levels of freestream turbulence in the core of the flow. The signature of the shock features becomes difficult to discern in the measurement planes closer to the side wall. This is because the levels of velocity fluctuations associated with the side wall boundary layer are much higher, masking the effects of the shock unsteadiness.

Another prominent feature that appears in the streamwise fluctuations near the spanwise centerline for the larger ramp (Figure 14a) is the elevated level of fluctuations associated with the shear layers downstream of the Mach stem. These features persist to the downstream limit of the measurement domain, and they mark the boundary between the wake and the surrounding faster fluid. Interestingly, the vertical distance between the top and bottom shear layers first decreases and then increases downstream of the Mach stem. This is consistent with the fact that the subsonic flow directly behind the Mach stem must pass through a minimum area sonic throat before being reaccelerated to supersonic speed. This minimum area occurs at around $x \approx 30 \mathrm{~mm}$.

For both test geometries, a region of high turbulence intensity develops on the top wall upstream of the foot of the compression ramp and continues along its length. These fluctuations are associated with the thickening and separation of the boundary layer in this region of severe adverse pressure gradient as well as any unsteadiness in the position of the shock foot. The extent of the high turbulence intensity region is significantly larger for the larger ramp geometry; however the peak levels of turbulence intensity are comparable in both cases. As the side wall is approached, the size of the high turbulence region decreases and is confined to an area closer to the top wall, and the maximum level of turbulence intensity decreases. This is consistent with the lesser degree of boundary layer thickening observed in the mean velocity plots (Figures 9 and 10) as the measurement planes approach the side wall. Along the top wall downstream of the compression ramp where the expansion fan reaccelerates the boundary layer, the streamwise turbulence intensity drops significantly but the magnitude of the vertical fluctuations remains relatively constant. From the full-field data in the larger ramp geometry case (Figures 14, 16, and 18), it is evident that the top wall boundary layer does not recover downstream of the compression ramp prior to the impingement of the reflected shock wave on the top wall.

On the bottom wall in the incident/reflected SBLI, there is a region of high intensity streamwise fluctuations, particularly near the spanwise centerline of the duct (Figures 13a and 14a). The trends in the velocity fluctuations in the incident shock interaction region across the span are similar between the two test cases but far more pronounced for the larger ramp geometry, so the discussion focuses primarily on the $h_{\text {ramp }} / \delta_{0}=0.93$ case. The region of highest intensity streamwise fluctuations is confined to the area below the Mach stem between the separation shock and the projected impingement point of the transmitted shock. The locus of maximum streamwise velocity fluctuations starts near the foot of the separation shock and extends diagonally upward and away from the bottom wall, then turns back down toward the bottom wall downstream of where the transmitted shock impinges on the separated zone. This is in qualitative agreement with the results of previous studies including Dupont et al. (2005), Humble et al. (2007), and Souverein et al. (2010). A more detailed analysis of the locus of maximum turbulence intensity throughout this interaction is given later in section 4.4. Downstream of the transmitted shock, the streamwise fluctuations in the accelerating boundary layer decay rapidly while the intensity of the vertical velocity fluctuations (Figure 16a) remains elevated. This means that the turbulence anisotropy varies significantly in the streamwise direction throughout the incident shock interaction, first increasing and then decreasing downstream of the transmitted shock. 

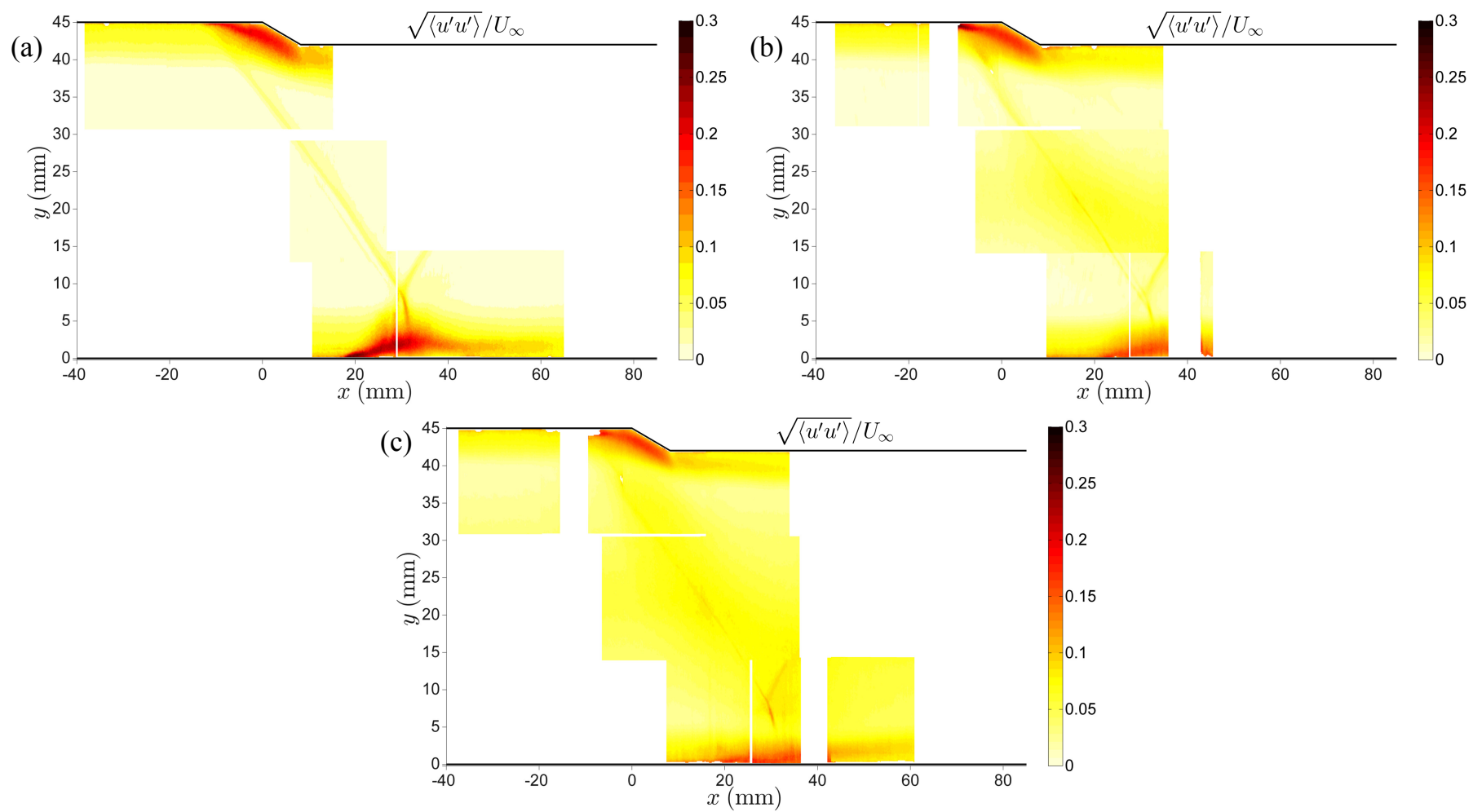

Figure 13: RMS of streamwise velocity fluctuations, normalized by $U_{\infty}$ for the mid-size ramp case $\left(h_{\text {ramp }} / \delta_{0}=0.56\right)$ in three planes across the tunnel span. (a) $z / \delta_{0}=3.89$ (near centerline), (b) $z / \delta_{0}=1.02$, and (c) $z / \delta_{0}=0.74$.
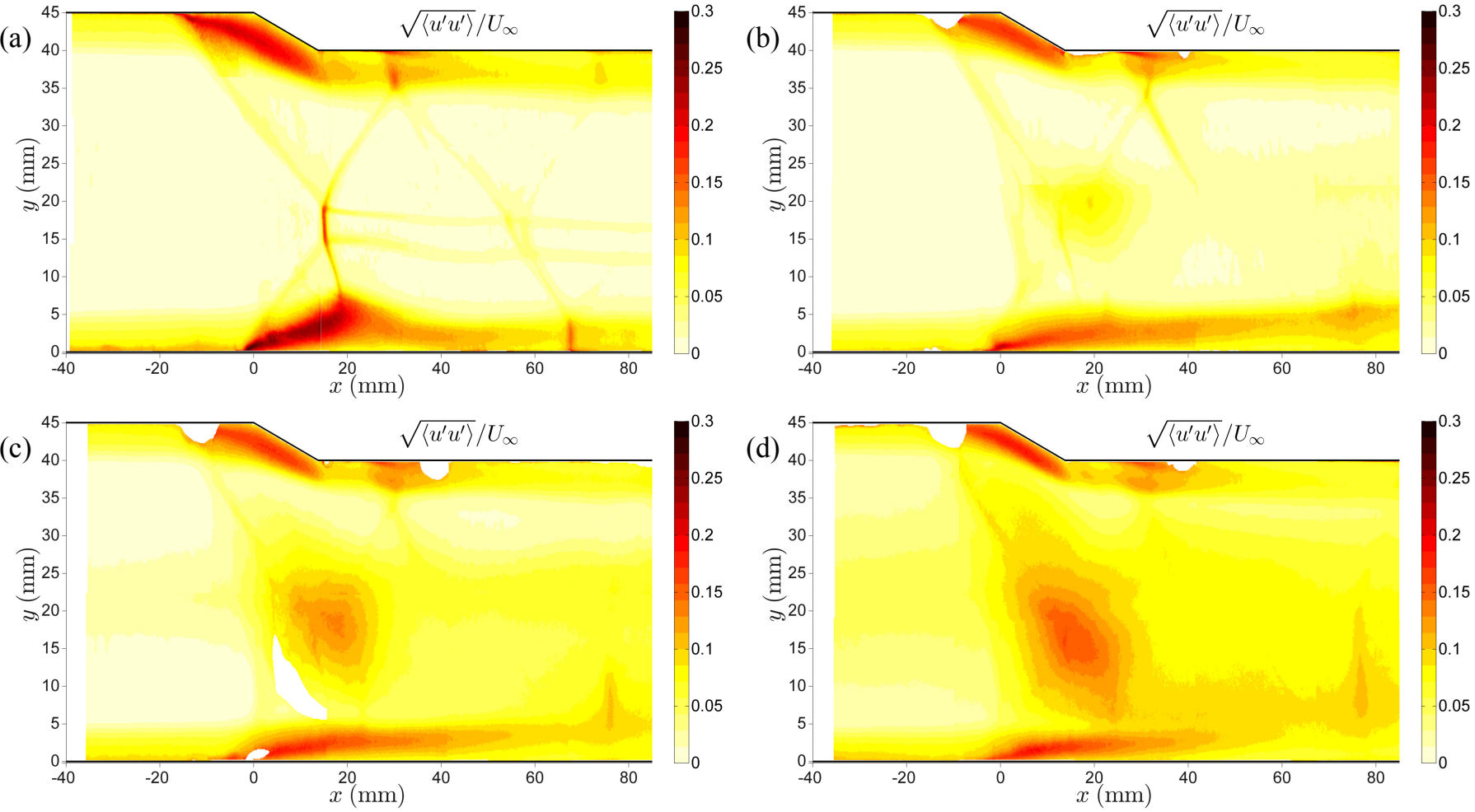

Figure 14: RMS of streamwise velocity fluctuations, normalized by $U_{\infty}$ for the largest ramp case $\left(h_{\text {ramp }} / \delta_{0}=0.93\right)$ in four planes across the tunnel span. (a) $z / \delta_{0}=3.89$ (near centerline), (b) $z / \delta_{0}=1.48$, (c) $z / \delta_{0}=1.02$, and (d) $z / \delta_{0}=0.74$. 

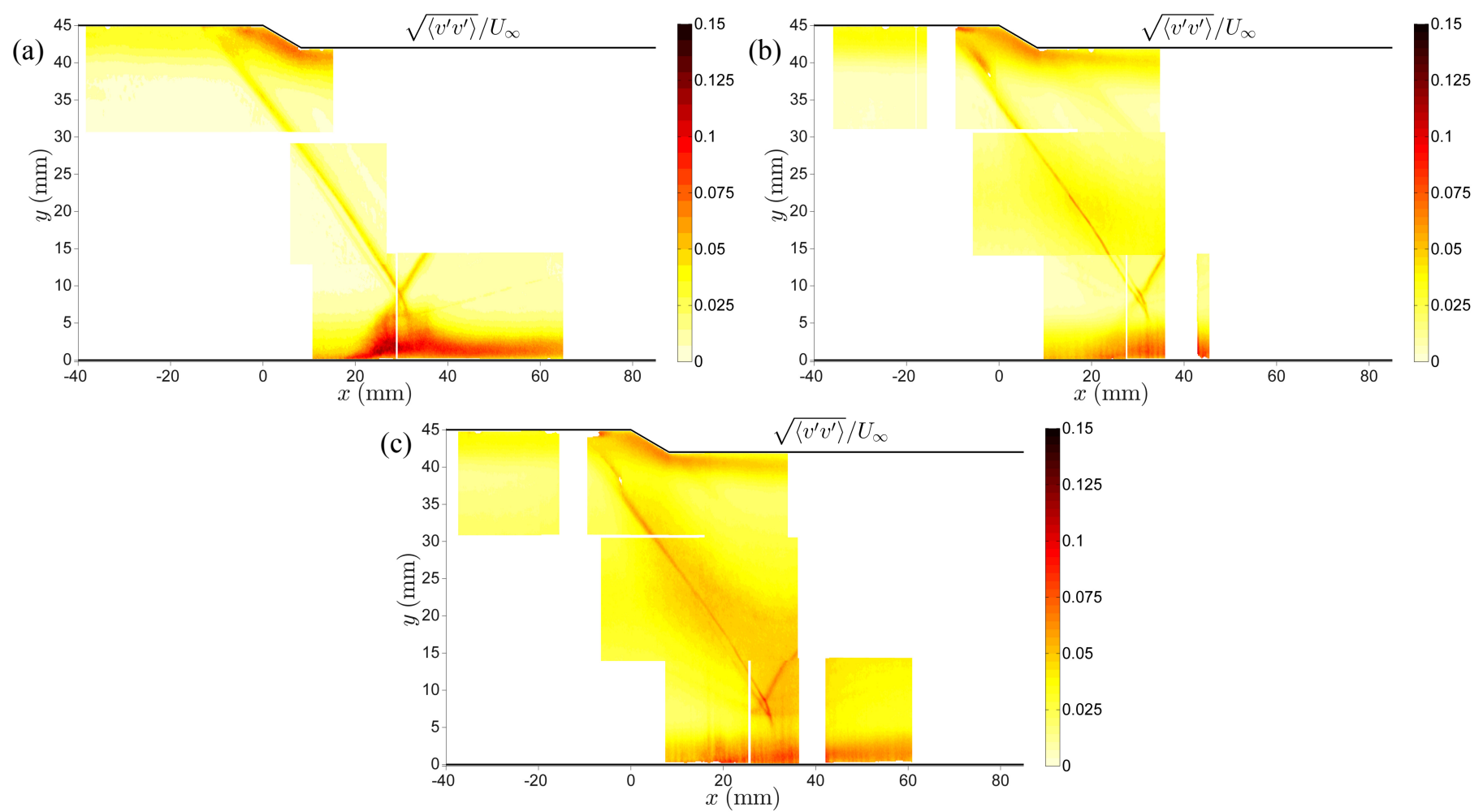

Figure 15: RMS of vertical velocity fluctuations, normalized by $U_{\infty}$ for the mid-size ramp case $\left(h_{\text {ramp }} / \delta_{0}=0.56\right)$ in three planes across the tunnel span. (a) $z / \delta_{0}=3.89$ (near centerline), (b) $z / \delta_{0}=1.02$, and (c) $z / \delta_{0}=0.74$.
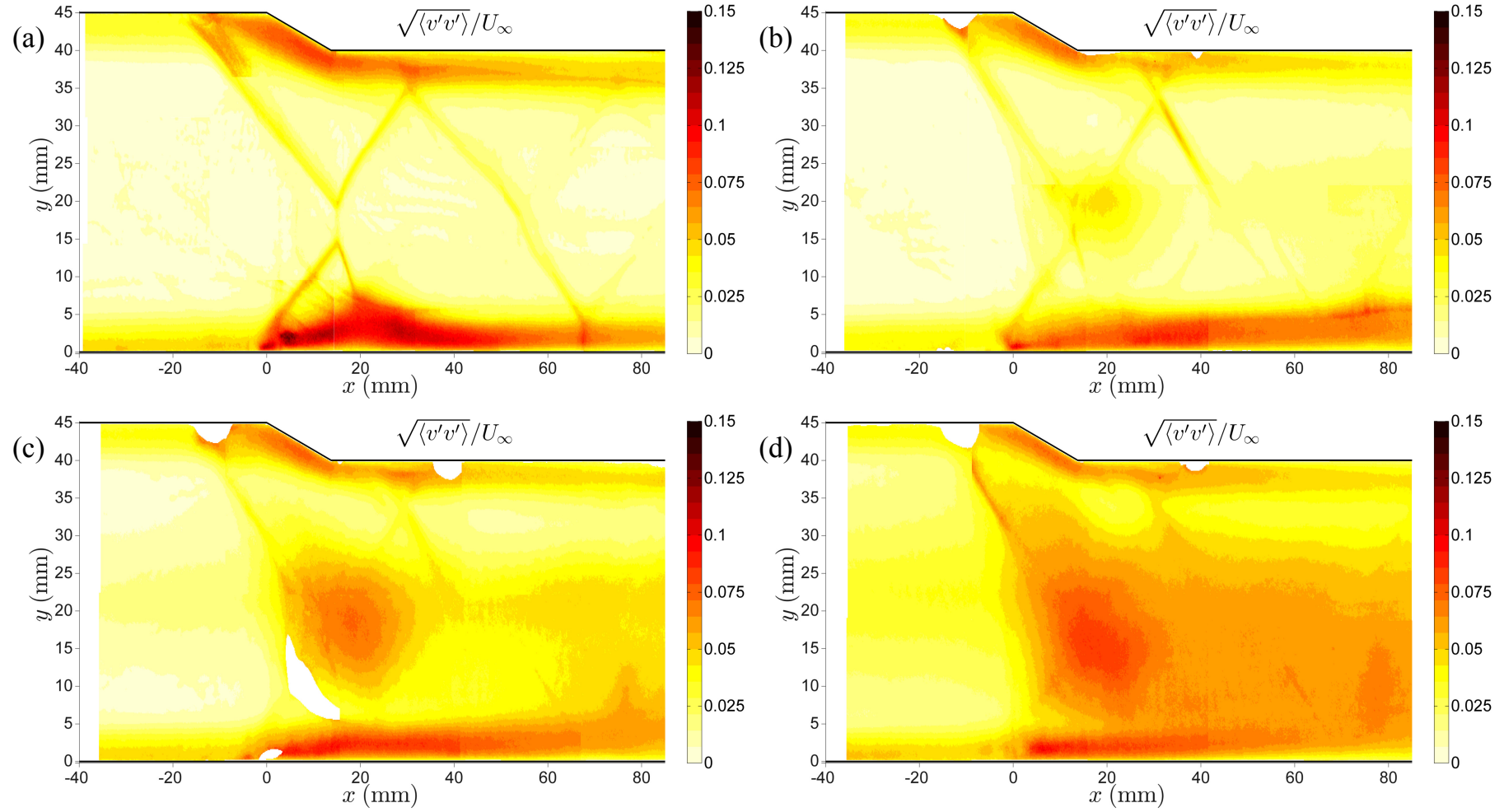

Figure 16: RMS of vertical velocity fluctuations, normalized by $U_{\infty}$ for the largest ramp case $\left(h_{\text {ramp }} / \delta_{0}=0.93\right)$ in four planes across the tunnel span. (a) $z / \delta_{0}=3.89$ (near centerline), (b) $z / \delta_{0}=1.48$, (c) $z / \delta_{0}=1.02$, and (d) $z / \delta_{0}=0.74$. 

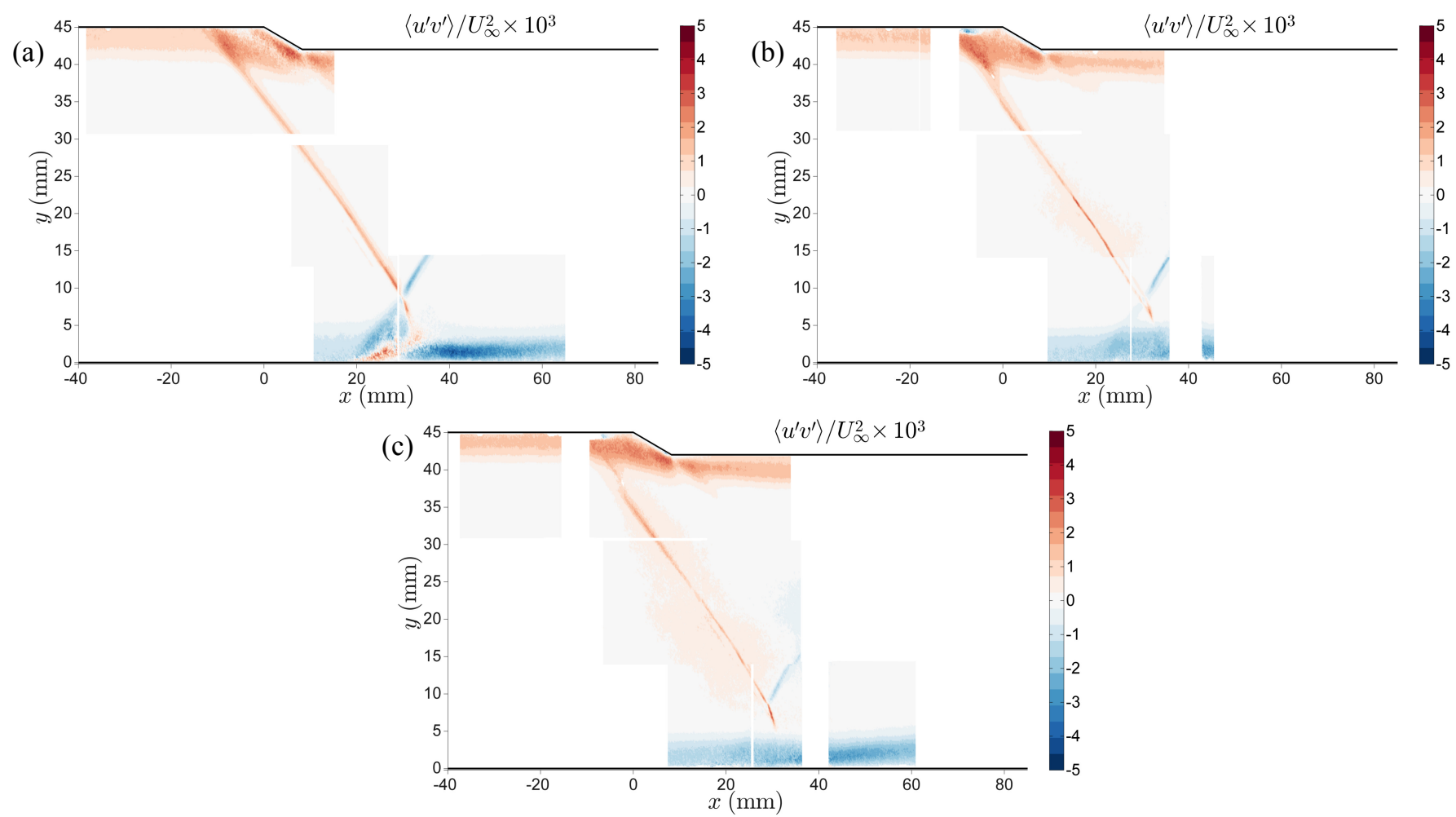

Figure 17: Reynolds shear stresses, normalized by $U_{\infty}^{2}$ for the mid-size ramp case $\left(h_{\text {ramp }} / \delta_{0}=0.56\right)$ in three planes across the tunnel span. (a) $z / \delta_{0}=3.89$ (near centerline), (b) $z / \delta_{0}=1.02$, and (c) $z / \delta_{0}=0.74$.
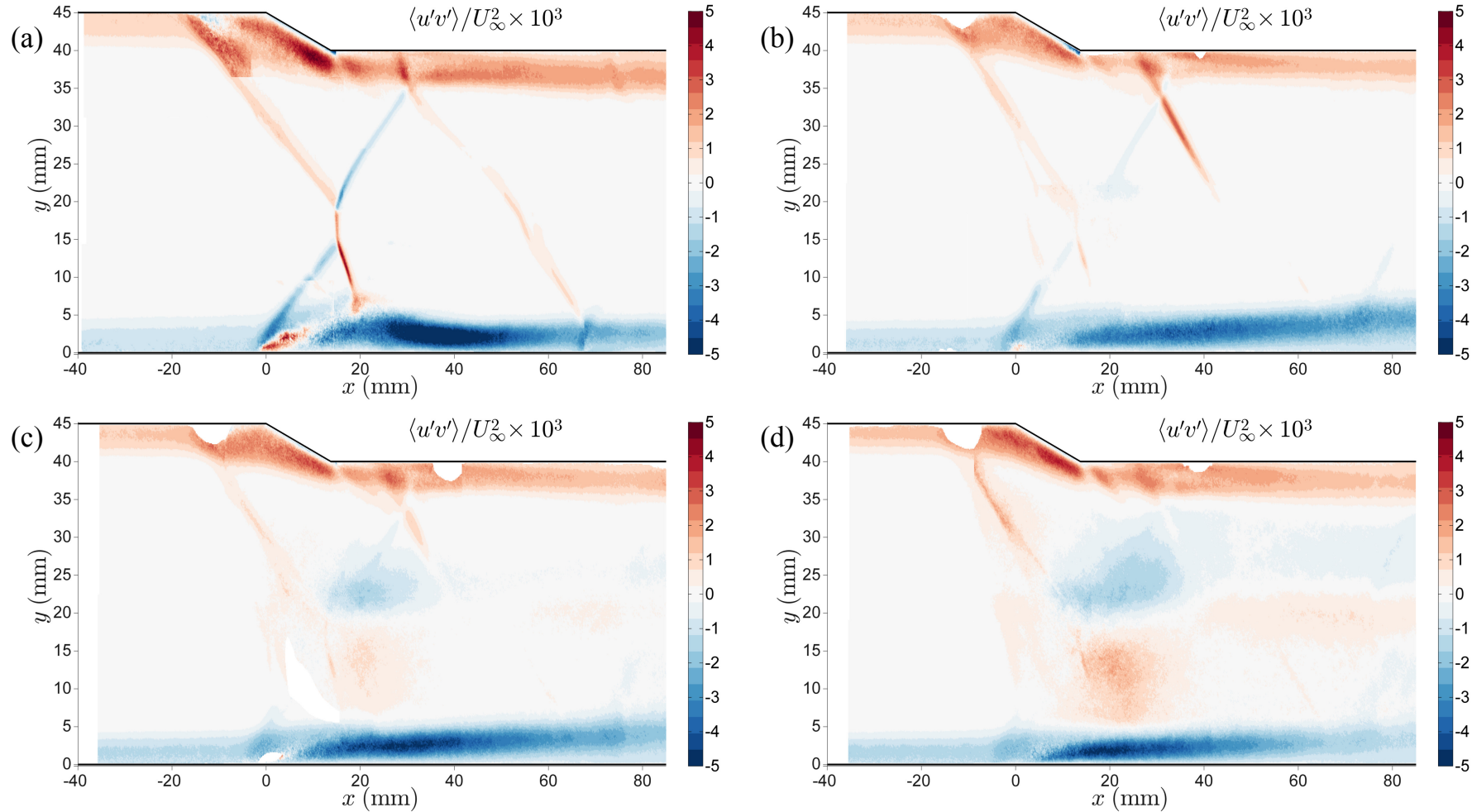

Figure 18: Reynolds shear stresses, normalized by $U_{\infty}^{2}$ for the largest ramp case $\left(h_{\text {ramp }} / \delta_{0}=0.93\right)$ in four planes across the tunnel span. (a) $z / \delta_{0}=3.89$ (near centerline), (b) $z / \delta_{0}=1.48$, (c) $z / \delta_{0}=1.02$, and (d) $z / \delta_{0}=0.74$. 
This behavior was noted by Ardonceau et al. (1980), and is attributed to the redistribution of the turbulent kinetic energy in the interaction, primarily through the pressure-strain correlation terms. For the larger ramp geometry near the spanwise centerline $\left(z / \delta_{0}=3.89\right)$, the ratio of $u^{\prime} / v^{\prime}$ just downstream of the separation shock is $\approx 6$, decaying to $\approx 2.9$ in the region between the separation and transmitted shocks, and dropping further to $\approx 1.3$ downstream of the transmitted shock.

In the planes closer to the side wall (Figures 14b-d and 16bd), the maximum observed levels of streamwise and vertical velocity fluctuations are lower than for the spanwise centerplane. For these off-center planes, the ratio of $u^{\prime} / v^{\prime}$ decreases everywhere in the flow except near the vertical centerline where the rapid thickening of the side wall boundary layer intensifies both $\sqrt{\left\langle u^{\prime} u^{\prime}\right\rangle}$ and $\sqrt{\left\langle v^{\prime} v^{\prime}\right\rangle}$. Furthermore, the locus of maximum streamwise and vertical velocity fluctuations in the $z / \delta_{0}=1.48$, 1.02 , and 0.74 planes occurs much closer to the bottom wall than in the spanwise centerplane. This observation is consistent with the fact that the thickening of the vertical boundary layer is less severe in these off-center planes.

Contours of Reynolds shear stress are given in Figures 17 and 18. In compressible flows, the Reynolds shear stress term includes the local mean density; however since density measurements are not available, we present representative measurements of $\left\langle u^{\prime} v^{\prime}\right\rangle$ instead. These contours clearly show the shock waves due to small oscillations in their positions. Positive values of $\left\langle u^{\prime} v^{\prime}\right\rangle$ are associated with shock waves that turn the flow downward (the incident and transmitted shocks) since negative fluctuations in the vertical velocity are correlated with deceleration in the streamwise direction. The opposite is true for shock waves that turn the flow upward. Toward the side wall boundary layer, the transmitted and reflected shock waves are no longer marked by sharp peaks in $\left|\left\langle u^{\prime} v^{\prime}\right\rangle\right|$. This is due to the thickening of the side wall boundary layer; the pressure signal is propagated through the large subsonic region, allowing the flow to decelerate and turn more gradually and effectively smearing out the effects in the streamwise direction (Figures 18c\&d).

The Reynolds shear stresses in the incoming boundary layers are positive on the top wall and negative on the bottom wall, and the magnitude of the fluctuations is amplified by the interaction of the vertical boundary layers with the incident and reflected shock waves. In particular, strongly negative values of $\left\langle u^{\prime} v^{\prime}\right\rangle$ are associated with the thinning of the bottom wall boundary layer downstream of the transmitted shock. This is consistent with the idea that a negative fluctuation in the vertical direction carries fluid with a higher streamwise momentum closer to the bottom wall, energizing and reaccelerating the boundary layer. This large streamwise-oriented region of high negative values of $\left\langle u^{\prime} v^{\prime}\right\rangle$ is also in qualitative agreement with the results of Humble et al. (2007) and Piponniau (2009).

Close to the bottom wall and directly downstream of the separation shock near the spanwise centerline, there is a coherent region of positive Reynolds shear stresses. This is interesting and counterintuitive because typically in a boundary layer positive values of $\left\langle u^{\prime} v^{\prime}\right\rangle$ are associated with negative values of $\partial U / \partial y$, which is not the case here. A region of oppositely signed $\left\langle u^{\prime} v^{\prime}\right\rangle$, though less prominent, is observed on the top wall in the compression ramp interaction directly downstream of the foot of the incident shock. The effect is seen in both test geometries, but it is more significant in the stronger interactions produced by the $h_{\text {ramp }} / \delta_{0}=0.93$ case. The region is oriented along the same direction as the locus of maximum $\sqrt{\left\langle u^{\prime} u^{\prime}\right\rangle}$ near the spanwise centerplane in both the compression ramp and incident shock interactions. A similar region of positive $\left\langle u^{\prime} v^{\prime}\right\rangle$ was observed by Humble et al. (2007) near the bottom wall downstream of the separation shock in an incident SBLI generated by an impinging shock with flow deflection of $8^{\circ}$ in a $M_{\infty}=2.1$ flow. The consistent presence of this feature in both geometries investigated in this study, combined with the similar observation by Humble et al. (2007) leads the authors to believe that the effect is physical; however the exact mechanism is unclear.

\subsection{Spanwise non-uniformities in incident SBLI}

In order to better highlight and quantify the non-uniformities across the span throughout the incident shock interaction for the $h_{\text {ramp }} / \delta_{0}=0.93$ test case, profiles of streamwise velocity and streamwise velocity fluctuations are plotted in Figures 19 and 20. The quantity $x^{*}=\left(x-x_{0}\right) / L_{\text {int }}$ describes a non-dimensional position within the interaction, where $x_{0}$ is the projected point of impingement of the separation shock on the bottom wall and $L_{\text {int }}$ is the interaction length, which is discussed in further detail in section 4.5. The profiles are plotted to a vertical height of $y / \delta_{0}=3$, which is contained below the Mach stem.

Figure 19 shows that the mean velocity profiles in the disturbed turbulent boundary layer develop an inflection point due to the strong adverse pressure gradient in the interaction zone. This behavior is observed both near the centerline and toward the side wall, though it is most obvious in the profiles at $z / \delta_{0}=3.89$. The most significant distortion and blockage occurs near the spanwise centerline of the duct, as denoted by the evolution of the severe velocity deficit in the profiles at $z / \delta_{0}=3.89$. The variation in the profiles across the span is most notable near $x^{*}=0.5$ where the maximum mean flow reversal occurs near the spanwise centerline. This streamwise location is also where the side wall boundary layer experiences its most severe thickening near the vertical centerline, causing the velocity profiles at $z / \delta_{0}=1.48,1.02$, and 0.74 to show a prominent non-monotonic behavior outside of $y / \delta_{0}=1$. It is remarkable to note how differently the profiles at $z / \delta_{0}=1.48$ and 3.89 evolve in the streamwise direction. Even though the $z / \delta_{0}=1.48$ measurement plane is well outside the side wall boundary layer in the inflow, it is heavily influenced by the thickening of the sidewall boundary layer throughout the interaction. At the end of the interaction length, the profiles collapse below $y / \delta_{0}=0.5$, but asymptote to different values far from the bottom wall.

The velocity fluctuation profiles in Figure 20 show that the fluctuations are amplified more near the spanwise centerline than closer to the side wall, particularly between $x^{*}=0.25$ and 0.5 . Outside of this streamwise region, there is fairly good agreement in the profiles across the span for $y / \delta_{0}<1$, with large discrepancies further from the bottom wall. Each profile shows a distinct a maximum in $\sqrt{\left\langle u^{\prime} u^{\prime}\right\rangle}$, associated with the separated shear layer in the incident/reflected shock interaction. The locus of max $\sqrt{\left\langle u^{\prime} u^{\prime}\right\rangle}$ is extracted from the PIV 


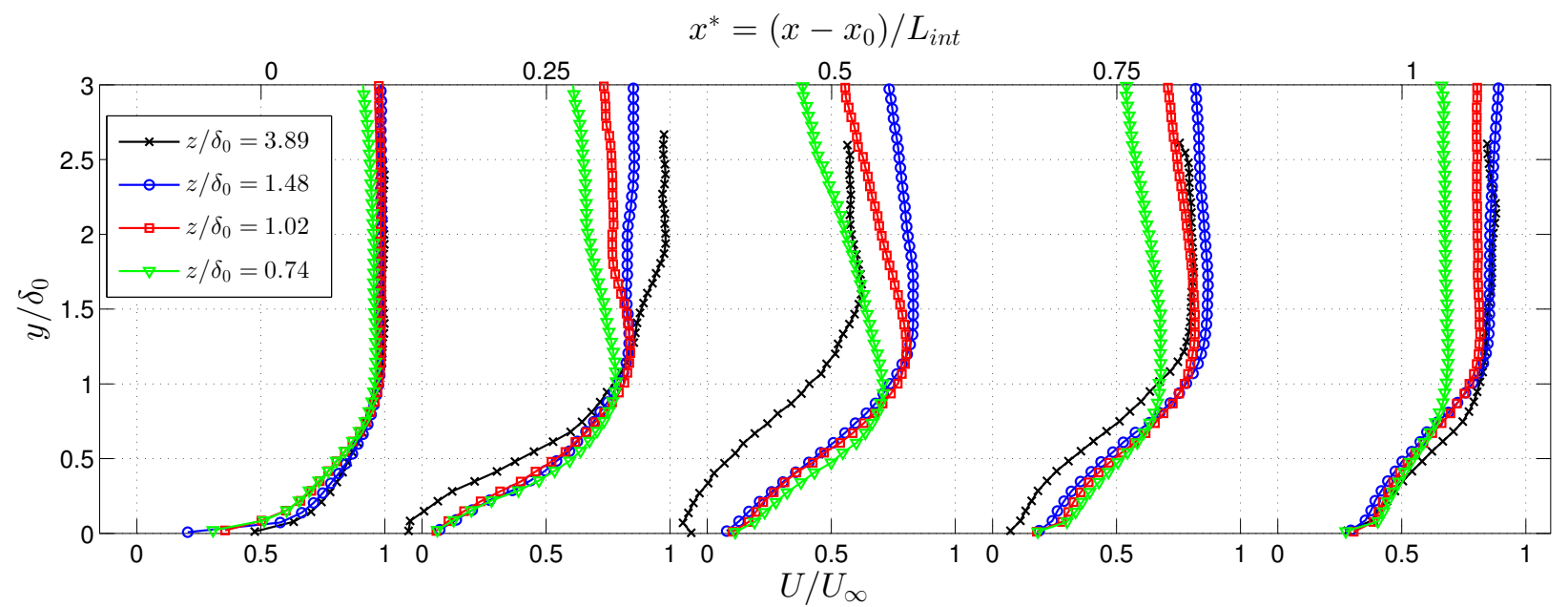

Figure 19: Streamwise evolution of the mean streamwise velocity profiles along the length of the incident shock interaction for the $h_{\text {ramp }} / \delta_{0}=0.93$ test case.

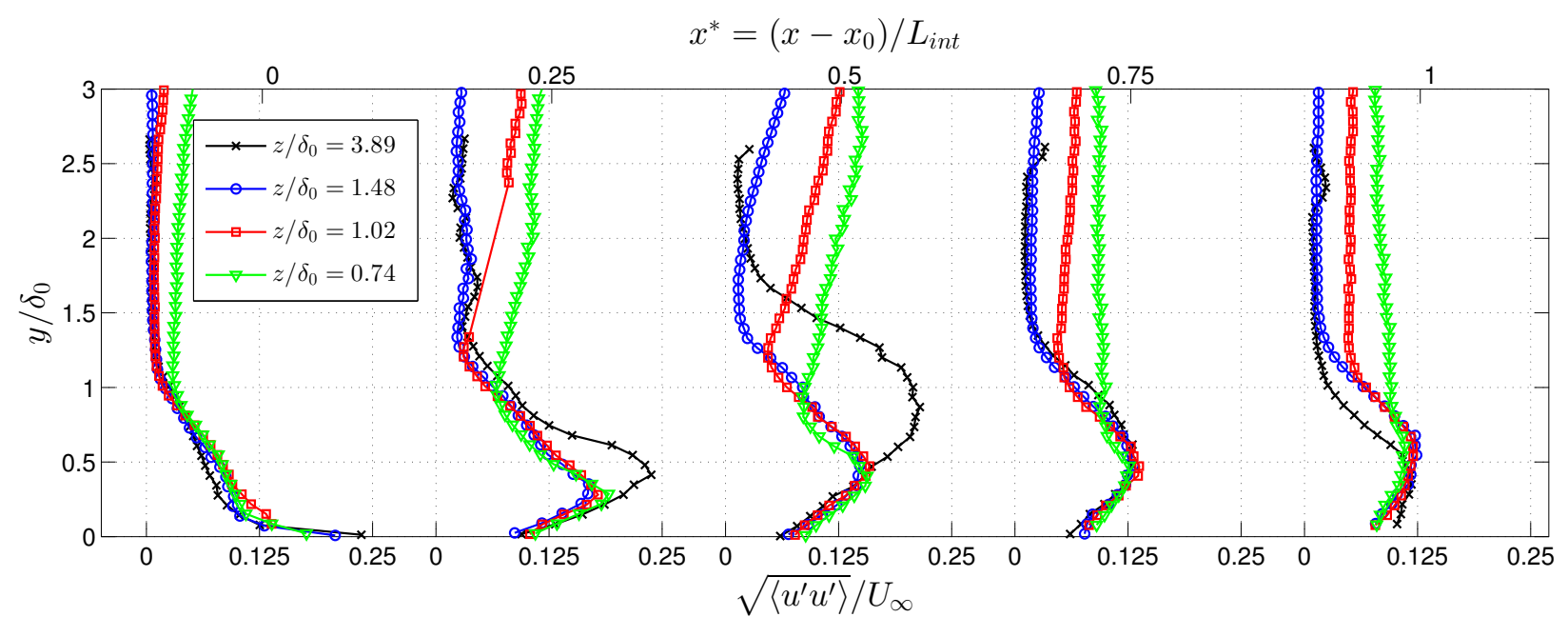

Figure 20: Streamwise evolution of the streamwise velocity fluctuation profiles along the length of the incident shock interaction for the $h_{\text {ramp }} / \delta_{0}=0.93$ test case.

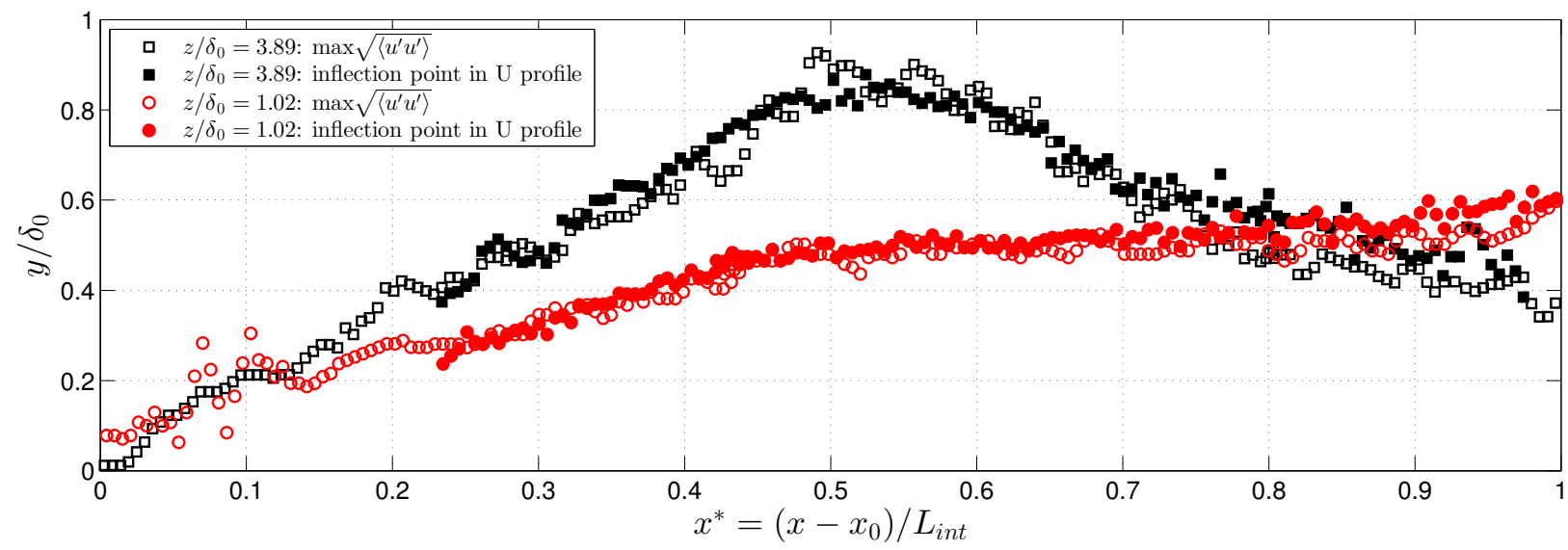

Figure 21: Locus of points corresponding to $\max \sqrt{\left\langle u^{\prime} u^{\prime}\right\rangle}$ and the inflection point of the $U$ profiles throughout the incident shock interaction for the $h_{\text {ramp }} / \delta_{0}=0.93$ case. The square symbols correspond to the the plane near the spanwise centerline $\left(z / \delta_{0}=3.89\right)$ and circles correspond to data at $z / \delta_{0}=1.02$. 
data in planes near the spanwise centerline $\left(z / \delta_{0}=3.89\right)$ and at the edge of the incoming undisturbed side wall boundary layer $\left(z / \delta_{0}=1.02\right)$, and plotted in Figure 21. First, it is interesting to note that the shapes of the loci of max $\sqrt{\left\langle u^{\prime} u^{\prime}\right\rangle}$ are quantitatively different across the span. Near the spanwise centerplane, there is a clear peak around $x^{*} \approx 0.5$ corresponding to the impingement point of the transmitted shock on the boundary layer; however closer to the side wall, the distance of $\max \sqrt{\left\langle u^{\prime} u^{\prime}\right\rangle}$ from the bottom wall increases monotonically throughout the interaction. Figure 21 also shows the loci of inflection points in the mean streamwise velocity profiles for the $z / \delta_{0}=3.89$ and 1.02 data planes. In both planes there is very good agreement between the location of the inflection point in the $U$ profile and the location of $\max \sqrt{\left\langle u^{\prime} u^{\prime}\right\rangle}$. Despite the fact that this flow is compressible and three-dimensional, these results agree with the classic behavior observed in a subsonic mixing layer (Bell and Mehta (1990)) or subsonic reattaching turbulent boundary layer (Eaton and Johnston (1981)).

\subsection{Spatial scales of the interactions}

One of the important spatial scales considered for shock boundary layer interactions is the interaction length, $L_{\text {int }}$. For SBLIs near compression ramps, this length is typically determined by the streamwise location upstream of the foot of the compression ramp where the mean wall pressure signal begins to rise. The interaction length can also be inferred by examining the mean streamwise velocity contours because the point where the wall pressure begins to rise corresponds directly to location where strong boundary layer thickening begins, or alternatively where $\partial U / \partial x$ begins rapidly decreasing, i.e. becoming more negative. In the data of Helmer et al. (2012), the mean wall pressure signal upstream of the compression ramp was measured. The location of the first noticeable pressure rise matches well with the location where the rate boundary layer thickening increases; both occur at location $\approx 1.4 \delta_{0}$ upstream of the foot of the compression ramp near the spanwise centerline. In the present experiments no pressure measurements were made, so the interaction lengths were determined using the PIV measurements alone. The interaction lengths associated with the compression ramp SBLI flow near the spanwise centerline are $L_{\text {int }} \approx 2.3 \delta_{0}$ and $L_{\text {int }} \approx 3.6 \delta_{0}$ for the mid-size $\left(h_{\text {ramp }} / \delta_{0}=0.56\right)$ and large $\left(h_{\text {ramp }} / \delta_{0}=0.93\right)$ ramp geometries, respectively. Previous arguments for the scaling of $L_{\text {int }}$ with the incoming Reynolds number, Mach number, boundary layer thickness, and compression ramp angle (see e.g. Délery and Marvin (1986)) assume large ramp geometries $\left(h_{\text {ramp }}>\delta_{0}\right)$ such that the size of the obstacle does not influence the interaction length. However, the results of the current experiments show that for smaller ramp sizes, the height of the ramp clearly also influences the interaction length.

In incident shock boundary layer interaction flows, the interaction length is related to the streamwise extent of the separated zone. Dupont et al. (2005) found that the length of the separated zone was well predicted by the distance between the projected impingement points of the incident and reflected shock waves on the bottom wall. Using this definition, the interaction lengths near the spanwise centerplane for the incident SBLIs are $L_{i n t} \approx 3.5 \delta_{0}$ and $L_{i n t} \approx 8.0 \delta_{0}$ for the mid-size and large ramps, respectively. Both of these interaction lengths are significantly larger than the value of $L_{i n t}=2.4 \delta_{0}$ for the incident SBLI in the $h_{\text {ramp }} / \delta_{0}=0.20$ investigated by Helmer et al. Because this method of determining $L_{i n t}$ is based on projected shock impingement points, both the shock angles and the vertical position of the intersection between the incident and reflected shocks play an important role. In the larger ramp case, the incident shock interacts with the expansion fan further downstream, leading to a stronger and larger separation, which corresponds to a shock crossing point further from the bottom wall. This effect is dominant over the steepening of the shock waves as the shock-generating ramp height increases (Table 4), which means that overall an increase in $L_{\text {int }}$ occurs as the shockgenerating ramp size increases.

Another way to quantify the size and strength of the incident shock interaction zone is to measure the length of the region over which mean flow reversal is observed on the bottom wall. This quantity does not correspond directly to the size of the separation bubble, but it provides valuable information for CFD validation, particularly given that accurately predicting the highly non-equilibrium separated boundary layer in this region is one of the biggest challenges to numerical simulations. The lengths associated with mean flow reversal along the bottom wall are $4.2 \delta_{0}, 0.71 \delta_{0}$, and 0 for the $h_{\text {ramp }} / \delta_{0}=0.93,0.56$, and 0.20 cases respectively.

Much work has been done to determine the frequency and length scales associated with the low frequency motion of the shock wave in SBLI flows (see Clemens and Narayanaswamy (2014) for review). The present experiments do not focus on identifying frequency content of the shock oscillation or the mechanism behind it, but we do compute the shock excursion length, $L_{e x}$, which is a measure of the streamwise distance traversed by the shock. As described by Dupont et al. (2008), the position of the shock wave can be inferred from any quantity which has a step change across the shock wave, such as pressure, density, or a component of velocity. We adopt the convention presented by Dupont et al. (2008) and Souverein et al. (2010) and use the vertical velocity across the oblique shock waves to determine $L_{e x}$. The median position of the shock wave corresponds to the location of $\max \sqrt{\left\langle v^{\prime} v^{\prime}\right\rangle}$. Then, the upstream and downstream extents of the shock oscillations are defined as the points corresponding to $\sqrt{\left\langle v^{\prime} v^{\prime}\right\rangle}=(1 / e) \max \sqrt{\left\langle v^{\prime} v^{\prime}\right\rangle}$. In these calculations, the low background level of freestream turbulence intensity is neglected. The process of determining $L_{e x}$ is illustrated in Figure 22. In the case of the nearly normal Mach stem, it is necessary to use the streamwise fluctuations, $\sqrt{\left\langle u^{\prime} u^{\prime}\right\rangle}$, instead of the vertical fluctuations as there is very little change in vertical velocity across this shock. It is important to note that this definition based on the velocity fluctuations does not allow $L_{e x}$ to be determined in the high turbulence region close to the walls, but it can be measured closer to the core of the duct.

Table 5 presents a summary of $L_{e x} / \delta_{0}$ for each of the major shock features measured near the spanwise centerplane of the channel. Here $y / \delta_{0}$ indicates the vertical position as which $L_{e x}$ was evaluated. In addition to the present cases, $L_{e x}$ has been extracted from the $h_{\text {ramp }} / \delta_{0}$ dataset of Helmer (2011) for com- 


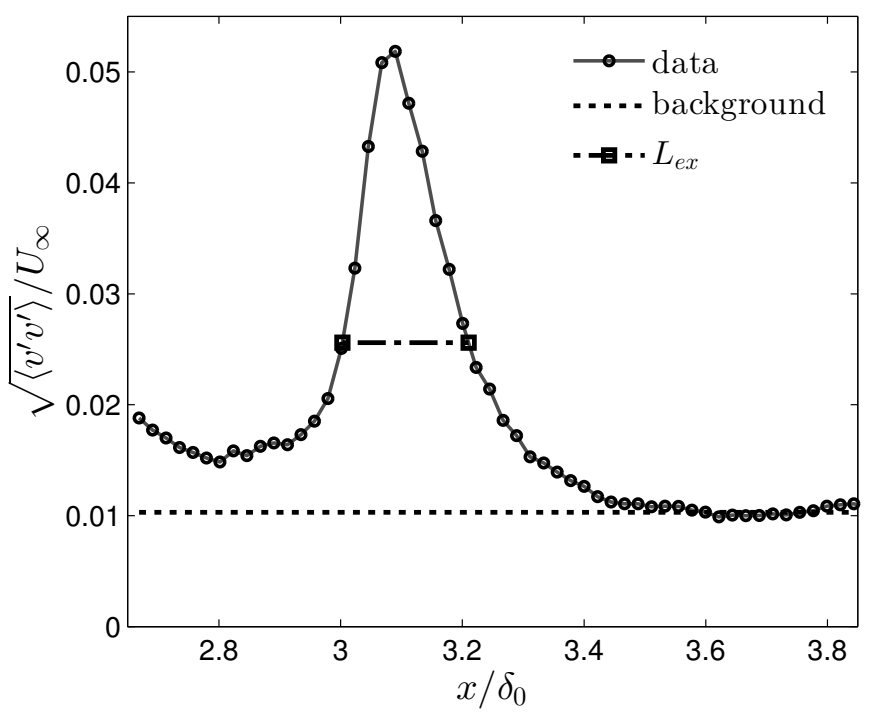

Figure 22: Streamwise evolution of $\sqrt{\left\langle v^{\prime} v^{\prime}\right\rangle} / U_{\infty}$ for illustrating the method for determining $L_{e x}$. Data are from the transmitted shock at a vertical location of $y / \delta_{0}=2.22$ in the $h_{\text {ramp }} / \delta_{0}=0.93$ test geometry.

Table 5: Shock excursion length, $L_{e x}$, measured for various shock structures near the spanwise centerplane $\left(z / \delta_{0}=3.89\right)$ for the $h_{\text {ramp }} / \delta_{0}=0.56$ and 0.93 test geometries. $L_{e x}$ was also extracted from the data of Helmer et al. for the $h_{\text {ramp }} / \delta_{0}=0.20$ for comparison.

\begin{tabular}{llll}
\hline \hline$h_{\text {ramp }} / \delta_{0}$ & shock type & $y / \delta_{0}$ & $L_{e x} / \delta_{0}$ \\
\hline \hline 0.20 (Helmer) & incident & 2.22 & 0.33 \\
\hline 0.20 (Helmer) & reflected & 2.22 & 0.37 \\
\hline \hline 0.56 & incident & 2.22 & 0.40 \\
\hline 0.56 & reflected & 2.22 & 0.38 \\
\hline \hline 0.93 & incident & 2.22 & 0.76 \\
\hline 0.93 & reflected & 4.07 & 0.36 \\
\hline 0.93 & separation & 2.22 & 0.50 \\
\hline 0.93 & transmitted & 2.22 & 0.22 \\
\hline 0.93 & Mach stem & 3.15 & 0.23 \\
\hline \hline
\end{tabular}

parison. For $h_{\text {ramp }} / \delta_{0}=0.2$ and 0.56 the only significant shock features within the measurement domain are the incident and reflected shocks. For the largest ramp case, there are additional shock features including the separation, transmitted, and Mach stem shocks as shown in Figure 8. $L_{e x}$ was evaluated for each of these using the same approach described above.

In general, the shock excursion lengths associated with all of the shock features are small relative to the incoming boundary layer size. The flow deflection across the incident shock wave ranges from $8^{\circ}$ for the smallest ramp geometry to $11.5^{\circ}$ for the largest ramp geometry. Dupont et al. (2006) report a value of $L_{e x} / \delta_{0}=0.58$ for the reflected shock outside the boundary layer $\left(y / \delta_{0}=3.45\right)$ for a similar interaction where the flow deflection angle across the incident shock wave is $9.5^{\circ}$ and the boundary layer is separated. This value is about a factor 1.5 larger than $L_{e x} / \delta_{0}$ for the reflected shock in all of the test cases presented in Table 5; however it is comparable to the value of $L_{e x} / \delta_{0}$ for the separation shock in the $h_{\text {ramp }} / \delta_{0}=0.93$ case. In their experiments, Dupont et al. (2006) also observed an increase in $L_{e x} / \delta_{0}$ of the reflected shock with increasing strength of incident shock wave. This trend is not seen in the current experiments; instead the value of $L_{e x} / \delta_{0}$ appears to be constant across the three test cases, regardless of the strength of the incident shock wave or the degree to which the bottom wall boundary layer is separated.

Furthermore, previous experimental studies by Humble et al. (2007) noted much larger values of velocity fluctuations associated with the reflected shock wave as compared to the incident shock wave. They attributed this to the larger degree of unsteadiness observed in the reflected shock wave. However, in the current study the peak values of the velocity fluctuations associated with the incident and reflected shock waves are approximately equal (see Figures 15 and 16), further supporting the conclusion that the reflected shock wave does not exhibit significant unsteadiness.

For the strongest interaction considered here $\left(h_{\text {ramp }} / \delta_{0}=\right.$ 0.93 test geometry), the least steady shock feature is the incident shock wave with $L_{e x} / \delta_{0}=0.76$. This indicates that the maximum excursion of the incident shock from its median position is about $\pm 2 \mathrm{~mm}$ in the streamwise direction. The transmitted shock and Mach stem are the steadiest features, with very low values of $L_{e x} / \delta_{0}$ of 0.22 and 0.23 . In dimensional terms, this means that any oscillations of these shocks about their median positions are limited to a region of less than $\pm 0.6 \mathrm{~mm}$ in the streamwise direction. We believe that the consistently low values of $L_{e x}$ for all of the shock features can be attributed to the very carefully controlled steady inflow conditions in the continuously operated wind tunnel facility.

While it would be interesting and informative to have measurements of the interaction and shock excursion length scales for the data planes away from the spanwise centerline of the duct, these values unfortunately cannot be inferred from the PIV measurements. The reason for this is that the techniques for calculating $L_{i n t}$ and $L_{e x}$ rely on identifying the shock features based on the elevated levels of velocity fluctuations in their vicinity. Closer to the side wall, the elevated levels of turbulence intensity associated with the side wall boundary layer and its interaction of the shock features obscures the signature of the shock waves.

\section{Conclusions}

SBLIs near a compression ramp and in an incident/reflected shock region were analyzed in detail for two low aspect ratio test configurations with differently sized shock-generating compression ramps. In the larger ramp configuration $\left(h_{\text {ramp }} / \delta_{0}=\right.$ 0.93), a Mach reflection occurred, and the incident shock interaction included a large region of mean flow reversal on the bottom wall close to the spanwise centerline of the duct. In the mid-size ramp geometry $\left(h_{\text {ramp }} / \delta_{0}=0.56\right)$, a regular reflection of the incident shock was observed and the overall size and strength of the interaction was significantly smaller, though 
larger than in the small ramp configuration $\left(h_{\text {ramp }} / \delta_{0}=0.20\right)$ investigated by Helmer (2011).

Throughout the incident shock interaction, the location of the maximum $\sqrt{\left\langle u^{\prime} u^{\prime}\right\rangle}$ was found to match well with the location of the inflection point in the mean velocity profiles. Despite the complexity and compressible nature of this flow, this is the same behavior observed in canonical subsonic boundary layer reattachment and mixing layer flows.

The relevant length scales of the various interactions and shock features near the spanwise centerline were extracted from the PIV measurements. The non-dimensional shock excursion lengths, $L_{e x} / \delta_{0}$, in these experiments were found to be small relative to other studies in the literature, indicating that the shock features in the present experiments exhibit less unsteadiness. Furthermore, for the reflected shock wave showed no dependence of $L_{e x} / \delta_{0}$ on the strength of the incident shock wave or degree of boundary layer separation was noted.

The experiments were designed and conducted specifically with the intent of using the resulting data for validation of CFD simulations of a complex three-dimensional non-equilibrium flow. High resolution PIV measurements were made over a large area encompassing multiple SBLIs and shock features. In order to gain physical insight into the three-dimensional structure of the flow and provide relevant data for validation, these measurements were repeated at multiple locations across the span, including one plane embedded inside the incoming side wall boundary layer.

Both the mean velocity and turbulence statistics indicate that the SBLIs in these low aspect ratio ducts are highly threedimensional, with steeper shock angles observed closer to the side wall than in the spanwise centerplane. In the case of the larger ramp geometry, there is no nominally two-dimensional region near the spanwise centerline. In order to accurately capture the effects imposed by the confinement and thickening side wall boundary layers, CFD simulations of these flows must include adequate treatment of all four walls of the duct. The detailed mean and turbulence measurements over a large region of the flow at several stations across the span of the duct provide physical insight into the shock and interaction structures, as well as a wealth of information for detailed CFD validation.

\section{Acknowledgements}

This research was funded by the US Department of Energy [National Nuclear Security Administration] under Award Number NA28614. Laura Campo is supported by the National Science Foundation Graduate Research Fellowship and the Gabilan Stanford Graduate Fellowship.

This issue honors Professor Brian Launder. While still a graduate student, I (JKE) had the opportunity to work with Professor Launder as the secretary to the Organizing Committee for the 1979/80 Stanford Conferences on Complex Turbulent Flows (the famous Kline's Olympics). Brian showed me great respect, listened to my opinions and gave feedback in a positive manner. As the acknowledged world leader in turbulence modeling, he pushed the notion that our community would be one of collaboration and friendship rather than ruthless competition. His ideas and approach played a significant role in shaping my early research agenda of experiments supporting the needs of modeling.

\section{References}

Adamson, T., Messiter, A., 1980. Analysis of two-dimensional interactions between shock waves and boundary layers. Annual Review of Fluid Mechanics 12 (1), 103-138.

Andreopoulos, J., Muck, K., 1987. Some new aspects of the shockwave/boundary-layer interaction in compression-ramp flows. Journal of Fluid Mechanics 180, 405-428.

Ardonceau, P., Lee, D., Alziary de Roquefort, T., Goethals, R., 1980. Turbulence behaviour in a shock wave/boundary layer interaction. In: AGARDCP-271, paper 8 .

Bell, J. H., Mehta, R. D., 1990. Development of a two-stream mixing layer from tripped and untripped boundary layers. AIAA Journal 28 (12), 2034-2042.

Beresh, S., Clemens, N., Dolling, D., 2002. Relationship between upstream turbulent boundary-layer velocity fluctuations and separation shock unsteadiness. AIAA Journal 40 (12), 2412-2422.

Bermejo-Moreno, I., Campo, L., Larsson, J., Bodart, J., Helmer, D., Eaton, J., 2014. Confinement effects in shock wave/turbulent boundary layer interactions through wall-modeled large-eddy simulations. Journal of Fluid Mechanics, in revision.

Bookey, P., Wyckham, C., Smits, A., 2005. Experimental investigations of Mach 3 shock-wave turbulent boundary layer interactions. In: 35th AIAA Fluid Dynamics Conference. Toronto, Ontario Canada, 6-9 June 2005.

Burton, D. M. F., Babinsky, H., 2012. Corner separation effects for normal shock wave/turbulent boundary layer interactions in rectangular channels. Journal of Fluid Mechanics 707, 287-306.

Christensen, K. T., 2004. The influence of peak-locking errors on turbulence statistics computed from PIV ensembles. Experiments in Fluids 36 (3), 484 497.

Clemens, N. T., Narayanaswamy, V., 2014. Low-frequency unsteadiness of shock wave/turbulent boundary layer interactions. Annual Review of Fluid Mechanics 46 (1), 469-492.

Davis, D., Gessner, F., 1989. Further experiments on supersonic turbulent flow development in a square duct. AIAA Journal 27 (8), 1023-1030.

Davis, D., Gessner, F., Kerlick, G., 1986. Experimental and numerical investigation of supersonic turbulent flow through a square duct. AIAA Journal 24 (9), 1508-1515.

Délery, J., Marvin, J., 1986. Shock-wave boundary layer interactions. Report, AGARDograph.

Dolling, D., Or, C., 1985. Unsteadiness of the shock wave structure in attached and separated compression ramp flows. Experiments in Fluids 3, 24-32.

Dolling, D. S., 2001. Fifty years of shock-wave/boundary-layer interaction research: what next? AIAA Journal 39 (8), 1517-1531.

Dupont, P., Haddad, C., Ardissone, J. P., Debiève, J. F., 2005. Space and time organisation of a shock wave/turbulent boundary layer interaction. Aerospace Science and Technology 9 (7), 561-572.

Dupont, P., Haddad, C., Debiève, J. F., 2006. Space and time organization in a shock-induced separated boundary layer. Journal of Fluid Mechanics 559, 255.

Dupont, P., Piponniau, S., Sidorenko, A., Debiève, J. F., 2008. Investigation by particle image velocimetry measurements of oblique shock reflection with separation. AIAA Journal 46 (6), 1365-1370.

Dussauge, J.-P., Dupont, P., Debiève, J.-F., 2006. Unsteadiness in shock wave boundary layer interactions with separation. Aerospace Science and Technology 10 (2), 85-91.

Eagle, W. E., 2012. An experimental study of three-dimensional inlet shockboundary layer interactions. Thesis, University of Michigan.

Eaton, J. K., Johnston, J. P., 1981. A review of research on subsonic turbulent flow reattachment. AIAA Journal 19 (9), 1093-1100.

Erengil, M., Dolling, D., 1991. Correlation of separation shock motion with pressure fluctuations in incoming boundary layer. AIAA Journal 29 (11), 1868-1877.

Gaitonde, D. V., Visbal, M. R., Shang, J. S., Zheltovodov, A. A., Maksimov, A. I., 2001. Sidewall interaction in an asymmetric simulated scramjet inlet configuration. Journal of Propulsion and Power 17 (3), 579-584. 
Galbraith, D., Turner, M., Orkwis, P., Weils, S., 2013. The effect of aspect ratio on a Mach 2.75 shock boundary layer interaction configuration. In: 51st AIAA Aerospace Sciences Meeting. Grapevine, TX, 7-10 January 2013.

Ganapathisubramani, B., Clemens, N. T., Dolling, D. S., 2007. Effects of upstream boundary layer on the unsteadiness of shock-induced separation Journal of Fluid Mechanics 585, 369.

Grilli, M., Hickel, S., Adams, N. A., 2013. Large-eddy simulation of a supersonic turbulent boundary layer over a compression/expansion ramp. International Journal of Heat and Fluid Flow 42, 79-93.

Hadjadj, A., Larsson, J., Morgan, B. E., Nichols, J., Lele, S. K., 2010. Largeeddy simulation of shock boundary-layer interaction. Proc. of CTR Summer Program, 141-152.

Hanada, T., Mitsuharu, M., Matsuo, K., 2005. Three-dimensional normal shock-wave/boundary-layer interaction in a rectangular duct. AIAA Journal 43 (10), 2182-2187.

Helmer, D. B., 2011. Measurements of a three-dimensional shock-boundary layer interaction. Thesis, Stanford University.

Helmer, D. B., Campo, L. M., Eaton, J. K., 2012. Three-dimensional features of a Mach 2.1 shock/boundary layer interaction. Experiments in Fluids 53 (5), 1347-1368.

Hornung, H., Robinson, M., 1982. Transition from regular to Mach reflection of shock waves, Part 2: The steady-flow criterion. Journal of Fluid Mechanics 123, 155-164

Hou, Y., Clemens, N., Dolling, D., 2003. Wide field study of shock induced turbulent boundary layer separation. In: 41st AIAA Aerospace Sciences Meeting. Reno, NV, 6-9 January 2003.

Humble, R. A., Elsinga, G. E., Scarano, F., van Oudheusden, B. W., 2009. Three-dimensional instantaneous structure of a shock wave/turbulent boundary layer interaction. Journal of Fluid Mechanics 622, 33.

Humble, R. A., Scarano, F., Oudheusden, B. W., 2007. Particle image velocimetry measurements of a shock wave/turbulent boundary layer interaction. Experiments in Fluids 43 (2-3), 173-183.

Hunter Jr., L. G., Reeves, B. L., 1971. Results of a strong interaction, wakelike model of supersonic separated and reattaching turbulent flows. AIAA Journal 9 (4), 703-712.

Ivanov, M., Vandromme, D., Fomin, V., Kudryavtsev, A., Hadjadj, A., Khotyanovsky, D., 2001. Transition between regular and Mach reflection of shock waves: new numerical and experimental results. Shock Waves 11, 199-207.

Lapsa, A., 2009. Experimental study of passive ramps for control of shockboundary layer interactions. Thesis, University of Michigan.

Loginov, M., Adams, N. A., Zheltovodov, A. A., 2007. Shock-wave system analysis for compression-decompression ramp flow. In: 5th International Symposium on Turbulence and Shear Flow Phenomena. Vol. 1. Munich, Germany, 27-29 August 2007, pp. 87-92.

Matheis, J., Budich, B., Hickel, S., 2013. Large eddy simulation of the transition process from regular to irregular shock-wave boundary-layer interaction. In: 8th International Symposium on Turbulence and Shear Flow Phenomena. Poitiers, France, 28-30 August 2013.

Piponniau, S., 2009. Instationnarités dans les décollements compressibles: cas des couches limites soumises à ondes de choc. Thesis, L'Université de Provence.

Pirozzoli, S., Bernardini, M., Grasso, F., 2010. Direct numerical simulation of transonic shock/boundary layer interaction under conditions of incipient separation. Journal of Fluid Mechanics 657, 361-393.

Ringuette, M. J., Bookey, P., Wyckham, C., Smits, A. J., 2009. Experimental study of a Mach 3 compression ramp interaction at $\operatorname{Re}_{\theta}=2400$. AIAA Journal 47 (2), 373-385.

Settles, G., Fitzpatrick, T., Bogdonoff, S., 1979. Study of attached and separated compression corner flowfields in high Reynolds number supersonic flow. AIAA Journal 17 (6), 579-585.

Settles, G. S., Dodson, L. J., 1994. Supersonic and hypersonic shock/boundarylayer interaction database. AIAA Journal 32 (7), 1377-1383.

Skews, B., 2000. Three-dimensional effects in wind tunnel studies of shock wave reflection. Journal of Fluid Mechanics 407, 85-104.

Souverein, L. J., Dupont, P., Debiève, J.-F., Van Oudheusden, B. W., Scarano, F., 2010. Effect of interaction strength on unsteadiness in shock-waveinduced separations. AIAA Journal 48 (7), 1480-1493.

Tieng, S., Chang, K., Yu, F., 1992. Holographic interferometric investigation of shock wave interaction with a ramp. Shock Waves 2, 133-138.

Touber, E., Sandham, N. D., 2009. Large-eddy simulation of low-frequency un- steadiness in a turbulent shock-induced separation bubble. Theoretical and Computational Fluid Dynamics 23 (2), 79-107.

Zheltovodov, A., 1996. Shock waves/turbulent boundary-layer interactions fundamental studies and applications. In: 27th AIAA Fluid Dynamics Conference. New Orleans, LA, 17-20 June 1996.

Zheltovodov, A., Maksimov, A., Shevchenko, A., Vorontsov, S., Knight, D., 1994. Experimental study and computational comparison of crossing shock wave-turbulent boundary layer interaction. In: International Conference on Methods of Aerophysical Research. Inst. of Theoretical and Applied Mechanics, Novosibirsk, Russia, August 1994. 